\title{
Firing and Cellular Properties of V2a Interneurons in the Rodent Spinal Cord
}

\author{
Kimberly J. Dougherty and Ole Kiehn \\ Mammalian Locomotor Laboratory, Department of Neuroscience, Karolinska Institutet, Stockholm S-171 77, Sweden
}

Previous studies have shown that a group of ventrally located neurons, designated V2a interneurons, play a key role in maintaining locomotor rhythmicity and in ensuring appropriate left-right alternation during locomotion (Crone et al., 2008, 2009). These V2a interneurons express the transcription factor Chx10. The aim of the present study was to characterize the locomotor-related activity of individual V2a interneurons, their cellular properties, and their detailed anatomical attributes in Chx10-GFP mice. A dorsal hornremoved preparation was developed to allow for visual whole-cell patch recordings from V2a interneurons along the entire lumbar spinal cord while at the same time leaving enough of the spinal cord intact to generate fictive locomotion. During drug-evoked locomotor-like activity, a large proportion of $\mathrm{Chx} 10$ cells showed rhythmic firing or membrane potential fluctuations related to either flexor or extensor activity in every lumbar segment. Chx10 cells received predominantly rhythmic excitatory input. Chx 10 neurons displayed a wide variety of firing and potential rhythmogenic properties. However, none of these properties was obviously related to the observed rhythmicity during locomotor-like activity. In dual recordings, we found no evidence of Chx10 neuron interconnectivity. Intracellular fills revealed diverse projection patterns with most $\mathrm{Chx} 10$ interneurons being local with projections to the central pattern generator and motor neuron regions of the spinal cord and others with long ascending and/or descending branches. These data are compatible with V2a neurons having a role in regulating segmental left-right alternation and ipsilateral motor neuron firing with little effect on rhythm generation.

\section{Introduction}

The spinal cord contains all of the essential neuronal circuitries needed to generate the coordinated motor output underlying locomotion. For limbed locomotion, the network components, including the neuronal circuits coordinating left-right and flexorextensor alternations and the rhythm-generating neurons that provide the excitatory drive required for locomotor activity, are all found within the spinal cord. Using electrophysiological studies, some of the basic organizational principles of the mammalian central pattern generator (CPG) are beginning to be unraveled (Gordon and Whelan, 2006; Kiehn, 2006; Clarac, 2008; Jankowska, 2008; Jordan et al., 2008; McCrea and Rybak, 2008; O'Donovan et al., 2008). The identification of molecular markers for classes of interneurons in the mouse spinal cord (Jessell, 2000; Goulding and Pfaff, 2005) has provided a new tool to identify and dissect the network structure of the locomotor CPG (Stepien and Arber, 2008). Four cardinal classes of ventrally located interneurons, called $\mathrm{V} 0-\mathrm{V} 3$, can be identified based on their expression of transcription factors during development. These classes display characteristic transmitter phenotypes and axonal projection pat-

Received Sept. 29, 2009; revised Nov. 3, 2009; accepted Nov. 6, 2009.

This work was supported by National Institutes of Health Grant R01 NS040795-08, a European Union grant (Spinal Cord Repair), Wings for Life, National Science Foundation Grant 0701166 (K.J.D.), the Swedish Medical Research Council, The Söderbergs Foundation, Hjärnfonden, and Friends of Karolinska Institutet. We thank Toshiaki Endo and Jesper Ryge for scripts and help with analysis in MatLab and R package, Ann-Charlotte Westerdahl and Margareta Widing for technical assistance, and Adolfo Talpalar for comments on a previous version of this manuscript.

Correspondence should be addressed to Ole Kiehn, Mammalian Locomotor Laboratory, Department of Neuroscience, Karolinska Institutet, Stockholm S-171 77, Sweden. E-mail: 0.kiehn@ki.se.

DOI:10.1523/JNEUROSCI.4821-09.2010

Copyright $\odot 2010$ the authors $\quad 0270-6474 / 10 / 300024-14 \$ 15.00 / 0$ terns. The V2a interneurons constitute a subpopulation of the V2 class and express Chx10. The Chx10 neurons are exclusively glutamatergic and project ipsilaterally (Al-Mosawie et al., 2007; Lundfald et al., 2007). Recently, we have shown that left-right alternation is disrupted in transgenic mice in which Chx10 neurons have been selectively ablated by diphtheria toxin $\mathrm{A}$, whereas alternation between flexors and extensors on the same side remains intact (Crone et al., 2008). In the absence of V2a interneurons, locomotor activity also showed an increase in the variability of burst frequency and amplitude (Crone et al., 2008). A consecutive study confirmed these findings and showed that the disruption in left-right alternation was most pronounced at intermediate locomotor frequencies (Crone et al., 2009). Together, these studies therefore suggested that V2a interneurons are acting on the commissural interneuron systems controlling left-right alternation (Butt and Kiehn, 2003; Quinlan and Kiehn, 2007) especially at intermediate locomotor frequencies, provide inputs to segmental motor neurons, and are not directly involved in rhythm generation (Crone et al., 2008, 2009). However, the previous studies did not provide any information about the relationship of the activity of the Chx10 population during locomotor-like activity.

Here, we use a transgenic mouse line that expresses enhanced green fluorescent protein (EGFP) driven by the promoter for Chx10 to record specifically from Chx10 neurons in a preparation that allows for the targeting of these cells along the entire lumbar spinal cord to study their locomotor-related activity, cellular properties, and connectivity. Our study shows that, during drug-induced locomotion, approximately two-thirds of the population of Chx10 neurons was rhythmically active, in phase with 
flexor or extensor motor neurons in all segments of the lumbar cord. Rhythmically active cells were driven by synaptic excitatory drive with little inhibitory input. The membrane properties of Chx10 neurons were heterogeneous and not clearly related to rhythmicity. Intracellular fills revealed diverse projection patterns with most Chx10 interneurons having local projections. Our data, together with data from an accompanying paper (Zhong et al., 2010), demonstrate that Chx10 neuron rhythmicity, synaptic input, and morphology are consistent with V2a neurons regulating segmental left-right alternation and ipsilateral motor neuron firing during locomotion and having little effect on rhythm generation.

Parts of this study have been presented in abstract form (Dougherty and Kiehn, 2008).

\section{Materials and Methods}

Chx10-EGFP transgenic mice from the Mutant Mouse Regional Resource Centers (MMRC, University of California, Davis, Davis, CA), created from a bacterial artificial chromosome on a FVB/N-Swiss Webster hybrid background strain, were used in all experiments. All experimental procedures followed the guidelines of the Swedish Animal Welfare Agency and were approved by the local Animal Care and Use Committee.

Mice [postnatal day 0 (P0) to $\mathrm{P} 5$ ] were anesthetized with isoflurane, decapitated, and eviscerated. A ventral laminectomy was performed and spinal cords were removed in a $\operatorname{cool}\left(4^{\circ} \mathrm{C}\right)$ low $\mathrm{Ca}^{2+}$ Ringer's solution, which contained the following (in $\mathrm{mm}$ ): $111 \mathrm{NaCl}, 3 \mathrm{KCl}, 11$ glucose, 25 $\mathrm{NaHCO}_{3}, 3.7 \mathrm{MgSO}_{4}, 1.1 \mathrm{KH}_{2} \mathrm{PO}_{4}, 0.25 \mathrm{CaCl}_{2}, \mathrm{pH}$ 7.4. The solution was aerated with $95 \% \mathrm{O}_{2} / 5 \% \mathrm{CO}_{2}$.

\section{Chx10 immunohistochemistry}

Dissected spinal cords from mice at P4 $(n=2)$ were immersion fixed in $4 \%$ paraformaldehyde, cryopreserved in $20 \%$ sucrose overnight, and stored at $-80^{\circ} \mathrm{C}$. Lumbar spinal cords were cryostat sectioned $(12 \mu \mathrm{m})$. Slides containing nonconsecutive sections were washed in PBS, blocked with $1 \%$ donkey serum for $1 \mathrm{~h}$, and incubated with 1:20,000 guinea pig anti-Chx10 (Thaler et al., 1999) at $4^{\circ} \mathrm{C}$ overnight. This antibody has been tested extensively for specificity (Thaler et al., 1999; Lundfald et al., 2007; Crone et al., 2008), specifically in that there is no cell staining when Chx10 neurons are ablated (Crone et al., 2008). Cy3 secondary antibodies (anti-guinea pig; Jackson ImmunoResearch) were used at 1:1000 and incubated for $1 \mathrm{~h}$. Fluorescence images were visualized on a Nikon Microphot-FX microscope. Images were overlaid in CorelDraw11, in which brightness and contrast were adjusted.

\section{Electrophysiological recordings}

Two different preparations were used for electrophysiological recordings. The first was a transverse slice preparation $(n=22$ mice) used for studies of cellular properties. After dissection, lumbar spinal cords were set in 2\% agar and vibratome (Microm; HM 650V) sectioned (250-300 $\mu \mathrm{m})$ transversely in a HEPES buffer solution, containing the following (in $\mathrm{mm}$ ): $101 \mathrm{NaCl}, 3.8 \mathrm{KCl}, 18.7 \mathrm{MgCl}_{2}, 25$ glucose, $1.3 \mathrm{MgSO}_{4}, 1.2$ $\mathrm{KH}_{2} \mathrm{PO}_{4}, 10 \mathrm{HEPES}, 1 \mathrm{CaCl}_{2}$, and continuously oxygenated with $100 \%$ $\mathrm{O}_{2}$. Slices were incubated in a normal Ringer's solution, containing the following (in mM): $111 \mathrm{NaCl}, 3 \mathrm{KCl}, 11$ glucose, $25 \mathrm{NaHCO}_{3}, 1.3 \mathrm{MgSO}_{4}$, $1.1 \mathrm{KH}_{2} \mathrm{PO}_{4}, 2.5 \mathrm{CaCl}_{2}, \mathrm{pH} 7.4$, aerated with $95 \% \mathrm{O}_{2} / 5 \% \mathrm{CO}_{2}$ for at least $30 \mathrm{~min}$ before recording.

The second preparation was a dorsal horn-removed preparation $(n=$ 23 mice) that allowed us to record from Chx10 neurons along the length of the lumbar (L1-L6) spinal cord during locomotor activity. For this, spinal cords extending from the lower thoracic spinal cord (Th8-Th10) to sacral segments were set in $2 \%$ agar. A single horizontal section from 375 to $550 \mu \mathrm{m}$ (depending on age of mouse and size of cord) was sliced from the dorsal horn along the entire length of the preparation using a vibratome. The ventral horn with roots attached was carefully dissected out of the agar and left to recover for at least $30 \mathrm{~min}$ in a regular Ringer's solution. A single ventral root recording was obtained using a suction electrode.
Locomotor-like activity was induced by bath application of NMDA $(5-10 \mu \mathrm{M})$ in combination with serotonin (5-HT; 5-10 $\mu \mathrm{M})$ and recorded with glass suction electrodes as ventral root activity (signal bandpass filtered, $100-1000 \mathrm{~Hz}$; gain, 5000-10,000) in lumbar segment 2 (L2), L3, L4, or L5. Dopamine (25-50 $\mu \mathrm{M})$ was also applied in addition to NMDA and 5-HT in some experiments. Dopamine did not alter the locomotor frequency $(0.16 \mathrm{~Hz}$ with dopamine, $n=10 ; 0.18 \mathrm{~Hz}$ without, $n=7 ; p=0.564$, Student's $t$ test). The main bursts in the L2 and L3 ventral roots reflect flexor activity, whereas the L4 and L5 ventral root activity reflects extensor activity (Whelan et al., 2000).

Both slices and dorsal horn-removed preparations were continuously perfused with aerated regular Ringer's solution at a flow rate of 4-5 $\mathrm{ml} / \mathrm{min}$. Patch electrodes were prepared from borosilicate capillary tubes ( $1.5 \mathrm{~mm}$ outer diameter, $0.86 \mathrm{~mm}$ inner diameter; Harvard Apparatus) using a two-stage puller (Narishige PP83), resulting in resistances of 5-9 M $\Omega$. Electrodes contained the following (in $\mathrm{mM}$ ): $128 \mathrm{~K}$-gluconate, 10 HEPES, $0.0001 \mathrm{CaCl}_{2}, 1$ glucose, $4 \mathrm{NaCl}, 5 \mathrm{ATP}, 0.3 \mathrm{GTP}, \mathrm{pH}$ 7.4. In some cases, $0.2 \%$ biocytin was included in the electrodes for cell reconstruction.

Whole-cell patch-clamp recordings were performed at room temperature using a Multiclamp amplifier (Molecular Devices). The signal was sampled at $10 \mathrm{kHz}$ and filtered at $6 \mathrm{kHz}$. Chx10-GFP cells were identified under fluorescence using a green fluorescent protein (GFP) filter (Olympus U-MW1B3) and patched using differential interference contrast optics at $60 \times$ (Olympus BX51WI scope). Cells were either recorded from one-by-one $(n=111)$ or at the same time as a nearby (electrode tips $<200 \mu \mathrm{m}$ apart) Chx10 cell (dual patch; $n=30$ pairs). Synaptic transmission was left unblocked both in experiments in which we assessed locomotor drug effects on Chx10 cells (slices and dorsal hornremoved preparations) and when we investigated the Chx10-toChx10 interactions.

For cellular properties, a series of current- and voltage-clamp protocols were performed in pClamp10 to obtain passive and active cellular properties. Input resistance was measured from hyperpolarizing steps in voltage clamp, and $\tau$ was obtained from the standard exponential fit of hyperpolarizing steps in current clamp, both using Clampfit. $C_{\mathrm{m}}$ was calculated from $R_{\mathrm{in}}$ and $\tau$ values above. Rheobase was the lowest current step at which the cell fired an action potential. Firing and rhythmogenic properties were obtained from long ( $1 \mathrm{~s}$ ) depolarizing and hyperpolarizing current pulses from a membrane potential of $-65 \mathrm{mV}$. Persistent inward currents were measured from voltage-clamp ramps from -80 to $-5 \mathrm{mV}$, ramped up at a speed of $30 \mathrm{mV} / \mathrm{s}$. The persistent inward current is seen as a negative slope region in the $I-V$ curve. To determine peak amplitudes and the onset of the negative slope region, ramps were leak subtracted based on the linear portion at the start of the ramp (Kuo et al., 2005). Values are reported as mean \pm SE. Membrane properties between groups were compared statistically using the Student's $t$ test or one-way ANOVA, depending on the number of groups being compared. The level of significance was $p<0.05$ for all statistical tests.

To look for synaptic and electrical coupling between Chx10 cells in dual recordings, 1-s-long current steps were performed in succession to "cell 1" while "cell 2" was held at $-65 \mathrm{mV}$ with bias current to avoid spiking. Additionally, short (10 ms) strong (150-300 pA) pulses were applied to cell 1 either singularly or in short trains of three or five pulses. The response of cell 2 was recorded, and an average of 50-100 trails was obtained. This procedure was then repeated from cell 2 to cell 1 .

In the dorsal horn-removed preparation, most recordings were from cells located in the same segment and on the same side of the cord (ipsilateral) as the ventral root being monitored. Intracellular data were acquired using the pClamp acquisition software Clampex (version 10; Molecular Devices). Membrane potentials were not adjusted for the liquid junction potential introduced by the whole-cell configuration (Kiehn et al., 1996; Raastad et al., 1998).

\section{Data analysis}

Locomotor analysis. The frequency (cycles per second; hertz) of the locomotor-like activity was calculated from 3-5 min of activity, taken at least 10 min after the initial burst of drug-induced activity, when the locomotor-like activity had stabilized. Locomotor-like activity was ana- 
lyzed using rectified and smoothed (time constant, $0.2 \mathrm{~s}$ ) signals of the ventral root activity in Spike2 (Cambridge Electronic Design). Cycle period $(P)$ was calculated as burst onset time to burst onset time. Quantification of the rhythmic activity in Chx10 neurons was done in two ways using circular plots and statistics (Kjaerulff and Kiehn, 1996; Tresch and Kiehn, 1999; Butt et al., 2002).

We first determined the degree of rhythmicity in individual Chx10 cells based on action potential firing during drug-induced locomotion. For this analysis, 25 spikes were selected randomly from at least 25 locomotor cycles. Onset of a locomotor burst in the ventral root corresponds to phase values of 0 in the circular plot, whereas the onset of the next burst corresponds to a phase value of 1 . The delay $(D)$ from the onset in the reference trace to spike in the Chx10 cell defines the phase value normalized to the reference period $(P)$. For individual experiments, the timings (phase value $=D / P$ ) of the 25 random spikes were plotted in a unitary circle at the time point of their occurrence in relation to the timing of ventral root burst. The mean timing of the 25 spikes was calculated and plotted as a vector in the circular plot (Zar, 1974). The length of the vector $(r)$ reflects the concentration of phase values around the mean timing. Raleigh's test (Zar, 1974) was used to determine whether the clustering of values reached statistical significance $(p<0.05)$. In this plot, if cells are firing in phase with the nearby ipsilateral ventral root their activity will span phase values from approximately around 0 to 0.5 . In some cases, Chx10 neurons were recorded on the contralateral side to the ventral root recording. For those cells, the points on the circular plots were rotated $180^{\circ}$ so that the phase value corresponded to the local ventral root. To determine spike tuning, normalized locomotor cycles were created from 25 consecutive locomotor cycles by dividing each cycle into 10 bins, and the number of spikes in each bin was plotted in a histogram (custom-made program in R package).

In some cells that were not spiking without current injection or in which spike timing was not found to have a significant direction (according to Raleigh's test), rhythmic oscillations in membrane potential were evident. Therefore, we next related the voltage fluctuations in the Chx10 neurons to the bursting in the nearby ventral root using circular statistics in a way similar to that described above for spiking cells. Here, 25 locomotor bursts were selected at random for analysis, with the ventral root as the reference trace and the intracellular recording as the test trace. The delay from the onset of the reference trace to the onset of the depolarizing voltage fluctuation in the intracellular trace was plotted in a unitary circular plot for each experiment. Resulting $R$ values from each cell were tested for significance ( $p<0.05$, Raleigh's test) and plotted in a circular plot. Depolarizing membrane oscillations that coincide with the ipsilateral segmental roots will have phase values that concentrate around the phase value of 0 , whereas depolarizing membrane oscillations that are out of phase with the ipsilateral segmental ventral roots will concentrate around the phase value of 0.5 .

Conductance measurements. To measure the synaptic inputs that Chx10 receive during locomotor activity, we used conductance measurements in accordance with previous published methods (Berg et al., 2007; Endo and Kiehn, 2008). Briefly, individual locomotor cycles were divided into 10 equal bins and the mean membrane potential was then obtained by averaging values from 5 to 10 locomotor cycles. This procedure was performed at two or three membrane potentials, and the total conductance was calculated for each bin as the slope of the current-voltage relationship obtained by linear regression. Decomposition of the conductances was based on the following equations:

$$
\begin{gathered}
G_{\text {total }}=G_{\mathrm{e}}+G_{\mathrm{i}}+G_{\text {leak }} \\
I_{\text {inj }}=G_{\mathrm{e}}\left(V_{\mathrm{m}}-E_{\mathrm{e}}\right)+G_{\mathrm{i}}\left(V_{\mathrm{m}}-E_{\mathrm{i}}\right)+G_{\text {leak }}\left(V_{\mathrm{m}}-E_{\text {leak }}\right),
\end{gathered}
$$

where $G_{\text {total }}$ is the total conductance, $G_{\mathrm{e}}$ is the excitatory conductance, $G_{\mathrm{i}}$ is the inhibitory conductance, $G_{\text {leak }}$ is the leak conductance, $I_{\text {inj }}$ is the injected current, $V_{\mathrm{m}}$ is the membrane potential, and $E_{\mathrm{e}}, E_{\mathrm{i}}$, and $E_{\text {leak }}$ are the reversal potentials for excitatory, inhibitory, and leak currents, respectively. To extract $G_{\mathrm{e}}$ and $G_{\mathrm{i}}$ from these equations, we first determined $G_{\text {total }}, I_{\text {inj }}$, and $V_{\mathrm{m}}$ as described above for each bin. $E_{\mathrm{i}}$ was set to the reversal for IPSPs with the intracellular solution used in the present study
$(-65 \mathrm{mV}) . E_{\mathrm{e}}$ was set to the commonly accepted value of $0 \mathrm{mV}$. Assuming that $G_{\text {leak }}$ and $E_{\text {leak }}$ were time- and voltage-independent, these parameters are set to arbitrary constant values. As previously described, we calculated the relative changes of $G_{\mathrm{e}}\left(\Delta G_{\mathrm{e}}\right)$ and $G_{\mathrm{i}}\left(\Delta G_{\mathrm{i}}\right)$ as a percentage of the mean total conductance.

\section{Biocytin processing}

Patch electrodes contained $0.2 \%$ biocytin. Recordings for successful reconstructions were 20-90 min in duration. Slices were left to incubate in the recording chamber for a minimum of 30-45 min (but often for several hours) after removal of the patch electrode. Dorsal horn-removed preparations remained in the recording chamber for the duration of the experiment and an additional $30 \mathrm{~min}$ after the last recording. Together, the prolonged in vitro staining procedures ( $50 \mathrm{~min}$ to $8 \mathrm{~h}$ ) should ensure diffusion of the biocytin to both dendritic and axonal processes. Sections or dorsal horn-removed preparations containing biocytin-filled cells were fixed in $4 \%$ paraformaldehyde overnight and then PBS at $4^{\circ} \mathrm{C}$. Sections were incubated in $1 \%$ avidin/biotin peroxidase complex solution in PBS-T for $3 \mathrm{~h}$, and then $0.01 \%$ diaminobenzidine tetrahydrochloride for $30 \mathrm{~min}$. Slices and dorsal horn-removed preparations were mounted on slides with $2 \%$ gelatin and dehydrated in graded ethanols and xylene. Cells were visualized on a light microscope, traced using camera lucida, scanned in, and retraced in CorelDraw11.

\section{Results}

\section{Chx10-GFP distribution in the spinal cord}

For all experiments, we used Chx10-EGFP transgenic mice, which express GFP in the hindbrain, spinal cord, and other areas of the CNS in which Chx10-positive cells have been described to be expressed (Rowan and Cepko, 2004; Cepeda-Nieto et al., 2005; Al-Mosawie et al., 2007; Lundfald et al., 2007; Crone et al., 2008) (Allen Institute for Brain Science, 2009; available from: http:// developingmouse.brain-map.org/). Here, we focus on the lumbar spinal cord (L2-L5) in which the CPG controlling hindlimb locomotion is localized (Kiehn and Kjaerulff 1998). GFPexpressing cells were visible along the entire length of the lumbar spinal cord and seen in two distinct regions in transverse sections of the lumbar spinal cord. The most brightly GFP-labeled cells were located in a band that extended medial-laterally from the central canal (lamina VII), with the central canal being approximately in the center of the band in the dorsal-ventral direction (Fig. $1 A, B$ ). This expression pattern was similar to what has been described for Chx10-positive cells in the postnatal lumbar spinal cord (Al-Mosawie et al., 2007; Lundfald et al., 2007; Crone et al., 2008). In the present mouse line, GFP expression was also found in dorsal horn neurons in the superficial laminae (laminae I-III). Here, GFP-positive cells were smaller and more tightly packed, and the GFP expression was weaker than in the ventral horn. At all levels of the lumbar spinal cord, there was a distinct separation between dorsal and ventral GFP-expressing cells.

As GFP expression may not always correctly report expression of the protein in transgenic mice (Hinckley et al., 2005; Wilson et al., 2005), we evaluated the coexpression of GFP and the Chx10 protein. A specific Chx10 antibody (Thaler et al., 1999; Lundfald et al., 2007; Crone et al., 2008) was used to verify Chx10-GFP expression. None of the dorsal horn cells was labeled with the Chx10 antibody. In contrast, GFP-expressing cells in the ventral population were all found to be labeled with the antibody and therefore Chx10 positive (Fig. $1 A, C-E$ ). Conversely, there were a few antibody-labeled cells in each section that did not contain GFP (Fig. 1, arrows).

From these experiments, we conclude that the neuronal GFP labeling in the ventral spinal cord reliably identifies Chx10positive cells. The ectopic GFP expression in the dorsal horn is clearly separated from the ventral cluster of GFP-positive cells 

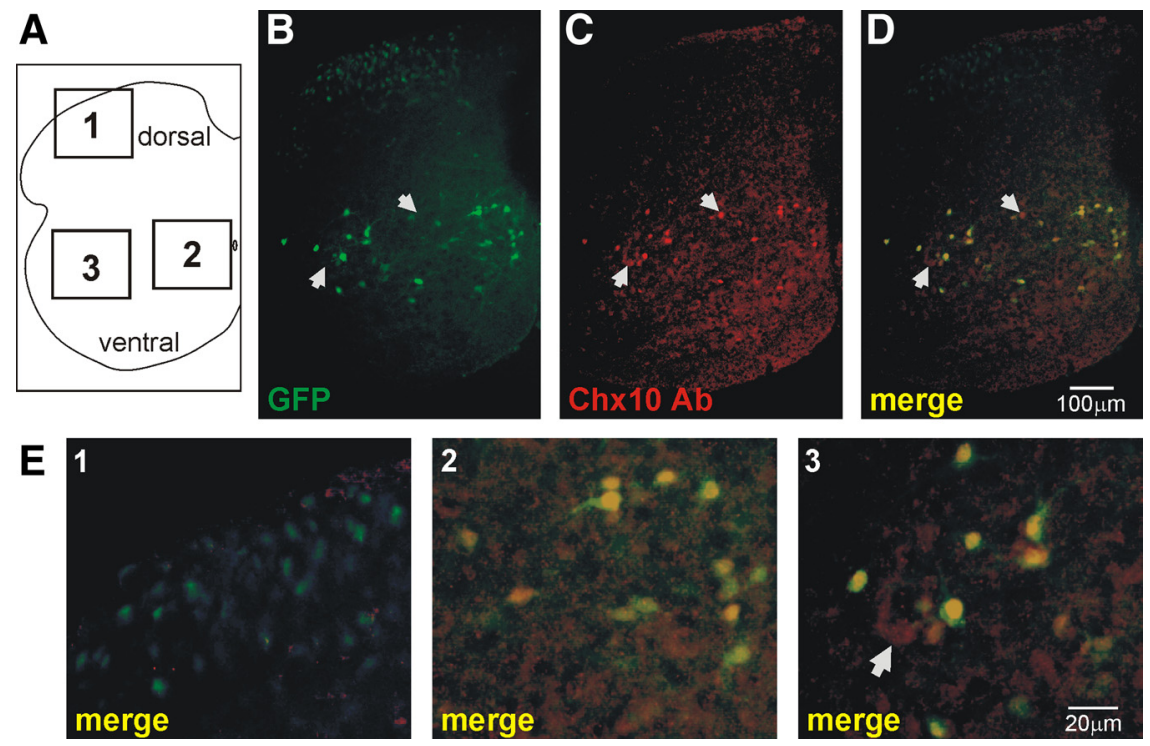

Figure 1. GFP is expressed in Chx10 neurons. $\boldsymbol{A}$, Schematic showing the position of the hemicord of the transverse section in $\boldsymbol{B}-\boldsymbol{D}$. The small boxes with numbers indicate the parts of the cord shown at higher resolution in $\boldsymbol{E}$. Dorsal and ventral horn are indicated. $\boldsymbol{B}$, Distribution of endogenous GFP fluorescence ( $g r e e n$ ) in a transverse section of lumbar spinal cord from a Chx10-EGFP mouse. Note that there is a dorsal and ventral population of GFP-positive neurons. C, Chx10 antibody labeling (red) in the same spinal section as in $\boldsymbol{B}$ shows that only the ventral population is labeled. $\boldsymbol{D}$, Overlay of antibody labeling (red) and endogenous fluorescence (green) show that all GFP-labeled cells in the ventral population are Chx10 positive (yellow). A few Chx10-positive cells were not GFP positive (arrows). $\boldsymbol{E}$, Higher resolution images of different locations of the spinal cord: dorsal horn $\left(\boldsymbol{E}_{\boldsymbol{1}}\right)$, medial in the ventral cord $\left(\boldsymbol{E}_{2}\right)$, and lateral in the ventral cord $\left(\boldsymbol{E}_{3}\right)$ (position indicated in $\boldsymbol{A}$ ). In the dorsal horn, GFP-positive cells were Chx10 negative $\left(\boldsymbol{E}_{1}\right)$, whereas in the ventral horn all GFP-positive neurons were Chx10 positive $\left(\boldsymbol{E}_{2}, \boldsymbol{E}_{3}\right)$. The arrow in $\boldsymbol{E}_{3}$ points to a Chx10 antibody-expressing cell that does not express GFP.

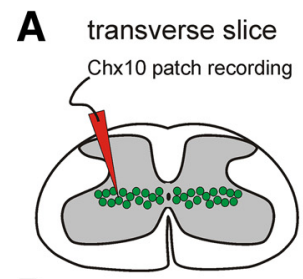

B

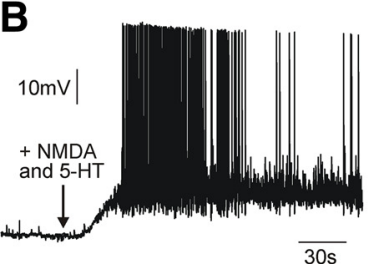

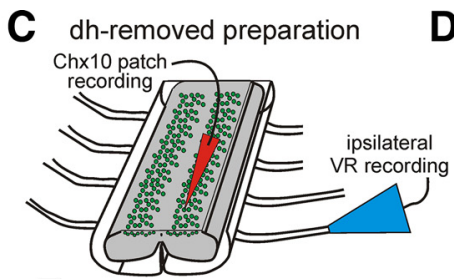

E

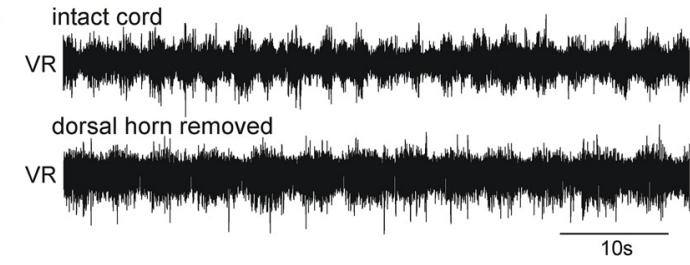

Figure 2. Experimental preparations. $\boldsymbol{A}$, Schematic of a transverse section of lumbar spinal cord with the approximate position of Chx10 neurons shown. $\boldsymbol{B}$, Response of Chx10 neuron recorded in transverse section to locomotor drugs (in this case, $5 \mu \mathrm{M}$ NMDA and $10 \mu \mathrm{m} 5-\mathrm{HT}$ ). Note the rapid depolarization. The arrow indicates when the drugs hit the perfusion chamber. $\boldsymbol{C}$, Schematic of the dorsal horn-removed preparation with the Chx10 neurons indicated. D, Map of the relative medial-lateral positions of Chx10 cells recorded in the dorsal horn-removed preparation. The dashed line indicates the midline, and VR denotes a ventral root. Note that this map is showing relative medial-lateral positioning of Chx10 cells. Cells were sampled throughout the lumbar (L2-L5) spinal cord but are all shown in one segment here. $\boldsymbol{E}$, Burst frequency of locomotor-like activity is reduced in the dorsal horn-removed prep (bottom) compared with the intact cord (top), even when the same drug concentrations are used (in this case, $5 \mu \mathrm{M}$ NMDA and $7 \mu \mathrm{m} 5-\mathrm{HT})$.

which makes it possible to safely target these latter cells in electrophysiological experiments. Below, we will call GFP-positive cells in the ventral cord Chx10 neurons.

\section{Chx10 neurons display rhythmic modulation during}

locomotor-like activity

In acute slices, whole-cell patch recordings were made from GFPexpressing cells in the ventral horn as indicated schematically in
Figure 2A. Application of locomotioninducing drugs typically resulted in a depolarization of the membrane potential (by $6.7 \pm 1.0 \mathrm{mV} ; n=14$ ) and an increase in spike firing and/or modulation of spontaneous firing (Fig. 2B). However, no obvious regular rhythm was seen in any cells recorded in slice.

To determine Chx10 cell activity in relation to locomotion, we therefore used a preparation in which the spinal cord dorsal to the central canal is removed by a single horizontal section (see Materials and Methods) (Fig. 2C). Thus, by removing the dorsal horn, GFP-expressing Chx10 neurons are close to the surface and visible for whole-cell patch recording (Fig. 2C). The remaining ventral part is sufficient to generate the locomotor rhythm (Kjaerulff and Kiehn, 1996), and locomotor-like activity can be monitored by recording from an intact ventral root. The dorsal horn was removed along the entire length of the lumbar spinal cord (L1-L6), which allowed us to sample Chx10 cells in segments in which either flexor (L2) or extensor locomotor (L5) activity dominates or segments in which flexor and extensor activity is more mixed (L3-L4) (Fig. 2D) (Cowley and Schmidt, 1994; Kiehn and Kjaerulff, 1996; Iizuka et al., 1997; Whelan et al., 2000).

Locomotor-like activity was measured by suction electrode recordings from single ventral roots (L2, L3, L4, or L5) and induced by application of NMDA, 5-HT, and, in some cases, dopamine. Activity was always induced first in the intact cord before washout and sectioning. This procedure increased the possibility to get reliable locomotion after sectioning the cord (for a similar procedure in hemicords, see also Hinckley et al., 2005). In general, the average frequency of locomotor-like activity was lower in the dorsal horn-removed preparation (mean, $0.17 \mathrm{~Hz}$ ) than in the intact cord (mean, $0.25 \mathrm{~Hz} ; n=17 ; p<0.05)$. In some cases, different concentrations of drugs, both higher $(n=5)$ and lower $(n=3)$ were used to elicit locomotion after removing the dorsal horn. However, this difference in frequencies of locomotor-like activity before and after cutting was also seen when the same concentrations of locomotioninducing drugs were used in the intact and dorsal horn-removed cords $(n=9)$ (Fig. $2 E$ ).

Cells recorded from in this preparation showed rhythmic activity. The degree of rhythmicity of Chx10 neurons was first quantified by determining the preferred firing phase of each Chx10 neuron with respect to the ipsilateral segmental ventral root bursting during drug-induced locomotion using circular statistics (Kjaerulff and Kiehn, 1996; Tresch and Kiehn, 1999; 
Butt et al., 2002). For each cell, 25 spikes were selected at random from at least 25 locomotor cycles. The timing of each spike, calculated as a phase value with respect to the onset of activity in the ventral root, was plotted in circular plot. Onset of a locomotor burst in the nearby ipsilateral ventral root corresponds to phase value of 0 in the circular plot, whereas the onset of the next burst corresponds to a phase value of 1 . In this plot, if cells are firing in phase with the nearby ipsilateral ventral root, their activity will span phase values from $\sim 0$ to 0.5 . If cells were recorded contralateral to the ventral root, phase values were rotated 0.5 so that firing was always plotted with respect to the ipsilateral ventral root. The mean timing of the 25 spikes was plotted as a vector in the circular plot in which the length of the vector $(r)$ reflects the concentration of phase values around the mean timing. Raleigh's test (Zar, 1974) was used to determine whether the clustering of values reached statistical significance $(p<0.05)$.

Of 62 recorded Chx10 neurons, 38 (61\%) fired action potentials (without current injection) during locomotor-like activity. Of the 38 spiking Chx10 neurons, $25(66 \%)$ were significantly rhythmic (Raleigh's test, $p<0.05$ ). Therefore, $40 \%$ of the total population of recorded Chx10 neurons was rhythmically firing. The degree of spiking in the rhythmically firing Chx10 neurons, as appreciated from the firing frequencies (Fig. $3 A-C$, top panels) and the normalized spike histograms (Fig. $3 A-C$, bottom right panels), varied substantially, ranging from relatively weak (Fig. 3A) and medium (Fig. 3B) to strong firing (Fig. 3C). Similarly to what has been observed for spike activity in rhythmically active interneurons in the rodent CPG area (Tresch and Kiehn, 1999; Raastad and Kiehn, 2000; Butt et al., 2002; Zhong et al., 2006), significantly rhythmically active Chx10 neurons showed broadly tuned spiking with spikes appearing throughout the cycle but at clearly preferred phase of firing (Fig. $3 A-C$, circular plots to the left and normalized spike histograms to the right). Figure $3 D$ shows the summary circular plot with vector end points plotted for rhythmically active Chx10 neurons (closed symbols) and nonrhythmically active Chx10 neurons (open symbols; the dashed inner circle indicates the significance level of $p=0.05$ ), recorded from segments L2/L3 (circles) and L4/L5 (triangles). From this phase plot, it can be recognized that Chx10 neurons along the cord fire both in phase $(0-0.5)$ and out of phase $(0.5-1)$ with the local ventral root, with concentrations around phase values of 0.25 and 0.75 . The dominating bursts during locomotor activity in the L2 and L3 ventral roots reflect flexor activity,
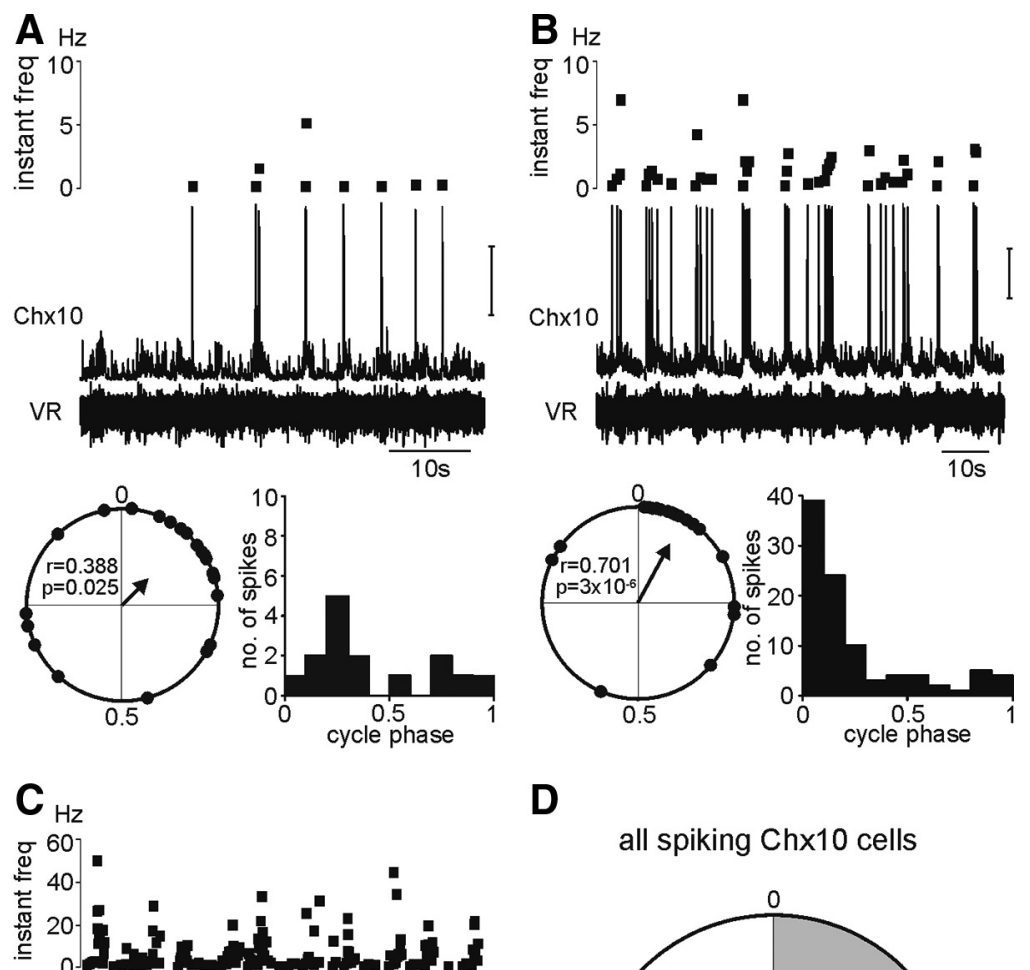

D

all spiking $\mathrm{Ch} \times 10$ cells

Figure 3. Chx10 neuron firing during locomotor-like activity. $A-C$, The degree of spiking in rhythmically firing neurons varied substantially, ranging from relatively weak $(\boldsymbol{A})$ and medium $(\boldsymbol{B})$ to strong firing $(\boldsymbol{C})$. This can be appreciated from the top graphs $(A-C)$ showing the instantaneous frequency (in hertz) plotted throughout the time course of the Chx10 neuron and ventral root (VR) recordings shown directly below and from the histograms (in the bottom right panels in $A-C$ ), which depict the number of spikes in 25 consecutive locomotor bursts and their position in a normalized locomotor cycle. Calibration: vertical (all), $20 \mathrm{mV}$. The circular plots shown for each neuron ( $\boldsymbol{A}-\boldsymbol{C}$, left bottom panel) were made from 25 random spikes taken from $25-60$ locomotor cycles. Note that all neurons shown have a preferred firing that is in phase with the local ventral root (vector is between 0 and 0.5 on the circular plot). The correspondence between circular plots and histograms may not be exact because spikes used for circular plots were selected at random from $>25$ cycles, whereas for the histograms all spikes from 25 consecutive cycles were used. D, Summary circular plot showing the phasing of all spiking Chx10 neurons ( 38 of 62 recorded). Each point on the plot corresponds to the vector value for a single cell. The length of the vector is a measure of the tuning, and the direction is the preferred firing. The dotted line represents significance at $p<0.05$. The circles indicate Chx10 neurons recorded in L2/L3, and the triangles indicate cells located in L4/L5. The filled symbols indicate rhythmic, and the open symbols indicate nonrhythmic cells. The shaded region of the circular plot is where cells firing in phase with the ipsilateral segmental root are located.

whereas the dominating bursts in the L4 and L5 ventral roots reflect extensor activity (Whelan et al., 2000), although intracellular studies of motor neuron activity during locomotor activity show that there are motor neurons in L2/L3 that are extensorrelated and motor neurons in L4/L5 that are flexor-related (Endo and Kiehn, 2008). Therefore, Chx10 neurons located in segments L2 and L3 that are in phase (phase values, $0-0.5 ; n=4$ ) in the circular plot are flexor-related and cells from segments L4 and L5 

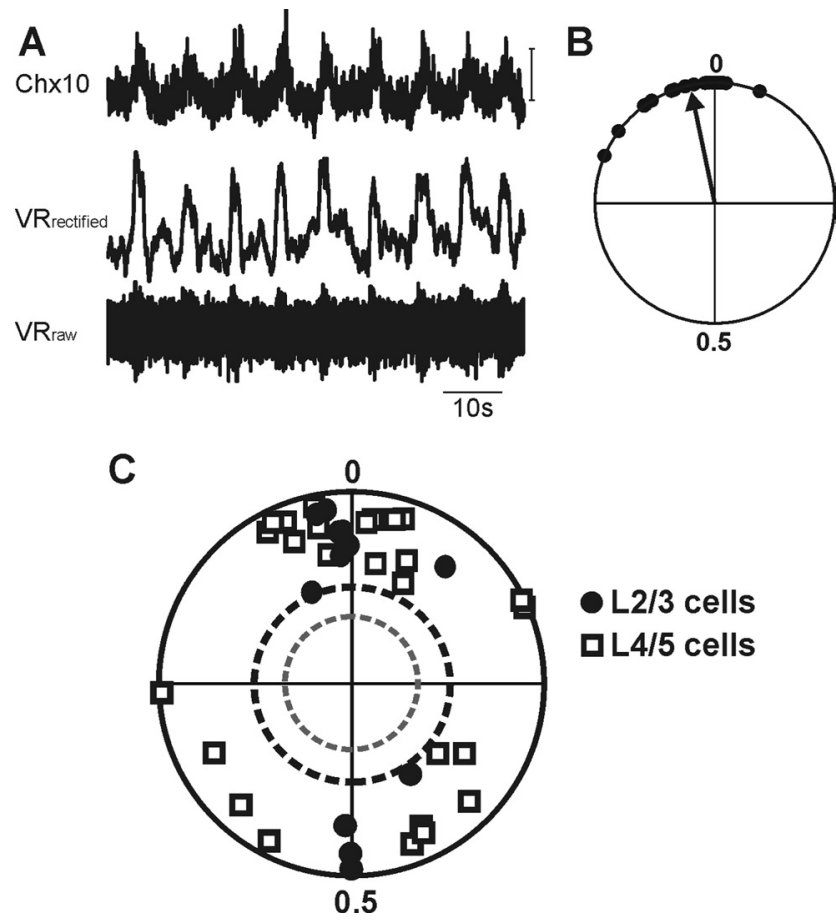

Figure 4. Locomotor-like rhythmic activity in Chx10 neurons. $\boldsymbol{A}$, Example of a Chx10 neuron with membrane oscillations in phase (top trace) with the local ventral root. The middle trace is rectified and smoothed $\left(V R_{\text {rectified }}\right)$, and the bottom trace is raw ventral root recording $\left(V R_{\text {raw }}\right)$. Calibration: $5 \mathrm{mV}$. $\boldsymbol{B}$, Circular plots corresponding to $\boldsymbol{A}$. Each point represents the onset of the membrane potential fluctuation as it relates to the onset of the ventral root burst (at 0 ). The arrows point to the preferred phase of the cell $(r=0.942 ; p<0.001)$. C, Summary circular plot of vectors from all recorded Chx10 neurons that showed rhythmic voltage oscillations $(n=40)$. The black circles indicate cells recorded in $\mathrm{L} 2$ and $\mathrm{L} 3$. The open squares indicate cells recorded in $\mathrm{L} 4$ and $\mathrm{L} 5$. The ventral root onset in the ipsilateral segmental root is 0 . The inner dashed circle (gray) indicates significance at $p<0.05$, and the outer dashed circle (black) denotes significance at $p<0.001$. Note that all cells are highly significantly rhythmic and that both L2/L3 and L4/L5 cells shows phase values in phase $(\sim 0)$ and out of phase $(\sim 0.5)$ with the ipsilateral segmental root.

that are in phase (phase values, $0-0.5 ; n=10$ ) are extensorrelated. Chx10 neurons in segments $\mathrm{L} 2$ and $\mathrm{L} 3$ that are active out of phase (phase values, $0.5-1.0 ; n=3$ ) with the local ipsilateral root are extensor-related, whereas out of phase L4/L5 Chx10 neurons $(n=8)$ will be classified as flexor-related. The combined plot of the preferred phases of firing therefore shows that Chx10 neurons fire both in flexor and extensor phases in the rostral and the caudal parts of the cord.

From the above analysis, we conclude that approximately two-thirds (25 of 38) of the spiking Chx10 neurons are rhythmically active based on spike timing. However, many of the nonrhythmic spiking cells ( 7 of 13 ) had obvious rhythmic membrane oscillations in the range of $2-10 \mathrm{mV}$ related to the ventral root bursting that were seen when cells were hyperpolarized below spiking threshold (but still more depolarized than $E_{\mathrm{Cl}}$ ). Similarly, in Chx10 neurons that were not spiking at rest, one-half (12 of 24) showed clear rhythmic membrane fluctuations related to the ventral root burst. Therefore, we next quantified the rhythmic activity in these additional groups, together with the rhythmically active neurons shown in Figure $3 D$, by relating their membrane potential oscillations to ventral root activity in normalized locomotor cycles (Fig. 4A). This was done by analyzing the phase relationship between the onset of the depolarization in the cell with respect to the onset of the ipsilateral segmental ventral root signal from 25 randomly selected cycles for each cell. The pre- ferred phase of activity analyzed in this way was plotted in a circular plot with the onset of the segmental ipsilateral ventral root activity having the phase value of 0 . The circular plot from the Chx10 cell shown in Figure $4 A$ is depicted in Figure $4 B$. The onset for the preferred phase of activity coincides with the onset of the ventral root activity. Figure $4 C$ summarizes the preferred phase of activity for all 40 of the Chx10 neurons that showed clear rhythmic voltage fluctuations during drug-induced locomotion, sorted with respect to whether they were recorded in L2/L3 or L4/L5. The majority of these neurons (26) were rhythmically active in phase, with the onset of the cellular depolarization occurring around the onset of the ipsilateral segmental ventral root burst (phase value, 0), in both flexor-related (Fig. 4C, 10 L2-L3 Chx10 neurons) and extensor-related (Fig. 4C, 16 L4-L5 Chx10 neurons) segments. Four Chx10 neurons in L2/L3 were rhythmically active out of phase (Fig. 4C) with the ipsilateral ventral root and therefore extensor-related, whereas 10 cells located in L4/L5 had phase values slightly before or after 0.5 (Fig. $4 C$ ), indicating that they were flexor-related. The phase values of L2 and L3 Chx10 neurons seemed to cluster more tightly around the phase values of 0 and 0.5 , indicating that they are more sharply tuned than the L4 and L5 Chx10 neurons. This relationship has also previously been determined for a larger group of L2 and L5 interneurons (Tresch and Kiehn 1999). The general stronger rhythmicity (as indicated by the larger $r$ values) observed when analyzing the membrane potential oscillations (Fig. 4C) compared with analyzing the rhythmic firing (in Fig. 3D) reflects the consistency of the timing of the onset of the membrane potential fluctuations, compared with the spike timing, with respect to the ventral root burst. As demonstrated by the examples in Figure 3, spikes did not only occur at the peak of the membrane potential fluctuation. Also, when there are many spikes (as for the cell in Fig. 3C), even if the frequency increases at the peak of a burst, a random sampling of 25 spikes will include spikes occurring between bursts, and therefore the $r$ value will decrease.

Together, the rhythmicity analysis showed that, based on spiking activity, $40 \%$ of the Chx10 neurons display rhythmic activity, whereas based on rhythmic voltage fluctuations, $65 \%$ of the Chx10 neurons are rhythmically active. Most cells are rhythmically active in phase with the ipsilateral ventral root whether it is flexor- or extensor-related (L2/L3 and L4-L5, respectively) but cells were also firing out of phase with the ipsilateral ventral root. Flexor and extensor activity is therefore represented in Chx10 cell rhythmicity in all segments of the lumbar cord. Since rhythmic fluctuations in membrane potential were evident in the majority of Chx10 neurons, we next analyzed the synaptic inputs to the Chx10 cells during locomotor-like activity.

\section{Excitatory synaptic drive dominates the rhythmic inputs to Chx10 neurons}

Using current-clamp recordings, previous studies of identified (Butt et al., 2002; Hinckley et al., 2005) and unidentified (Raastad and Kiehn, 2000) ventrally located interneurons have shown that the synaptic drive can be pure excitation, pure inhibition, or push-pull of alternating excitation and inhibition. Here, we use a continuous conductance measurement method (Berg et al., 2007; Endo and Kiehn, 2008) to reveal the synaptic inputs to Chx10 neurons. This method decomposes the conductance into a leak conductance, and synaptic excitatory $\left(G_{\mathrm{e}}\right)$ and inhibitory conductances $\left(G_{\mathrm{i}}\right)$ in normalized locomotor cycles divided into 10 equal-sized bins (for details, see Materials and Methods). Bins 1-5 correspond to the active phase of activity (in phase with the ventral root for in-phase-active cells and out of phase for out-of- 
phase-active cells), whereas bins 6-10 correspond to the inactive phase of activity. By relating the time of the synaptic conductance changes to the locomotor cycle, we determine the dominating rhythmic inputs to individual cells. The measurements show that the inputs received by Chx10 neurons were predominantly excitatory and that these excitatory inputs occurred in the active phase (Fig. $5 A$ ) and were sharply tuned (Fig. 5C). In contrast, inhibitory conductances show equal size throughout the locomotor cycle with no evident phasing (Fig. $5 B, D$ ).

To further verify that it is the excitation that leads to the rhythmic activity, a subset of Chx10 neurons that showed clear rhythmic depolarizations at potentials around -50 to $-40 \mathrm{mV}$ were hyperpolarized to membrane potentials ranging from -70 to $-80 \mathrm{mV}$, well below the reversal potential for chloride. Of these 22 cells, only one had membrane oscillations that reversed at hyperpolarized potentials indicative of rhythmic inhibitory drive.

We conclude that excitatory synaptic inputs drive the membrane oscillations seen in Chx10 cells with no or little contribution rhythmic inhibitory inputs. These findings fit well with our previous model, which has Chx10 cells receiving direct excitation from the rhythmgenerating neurons (Crone et al., 2008).
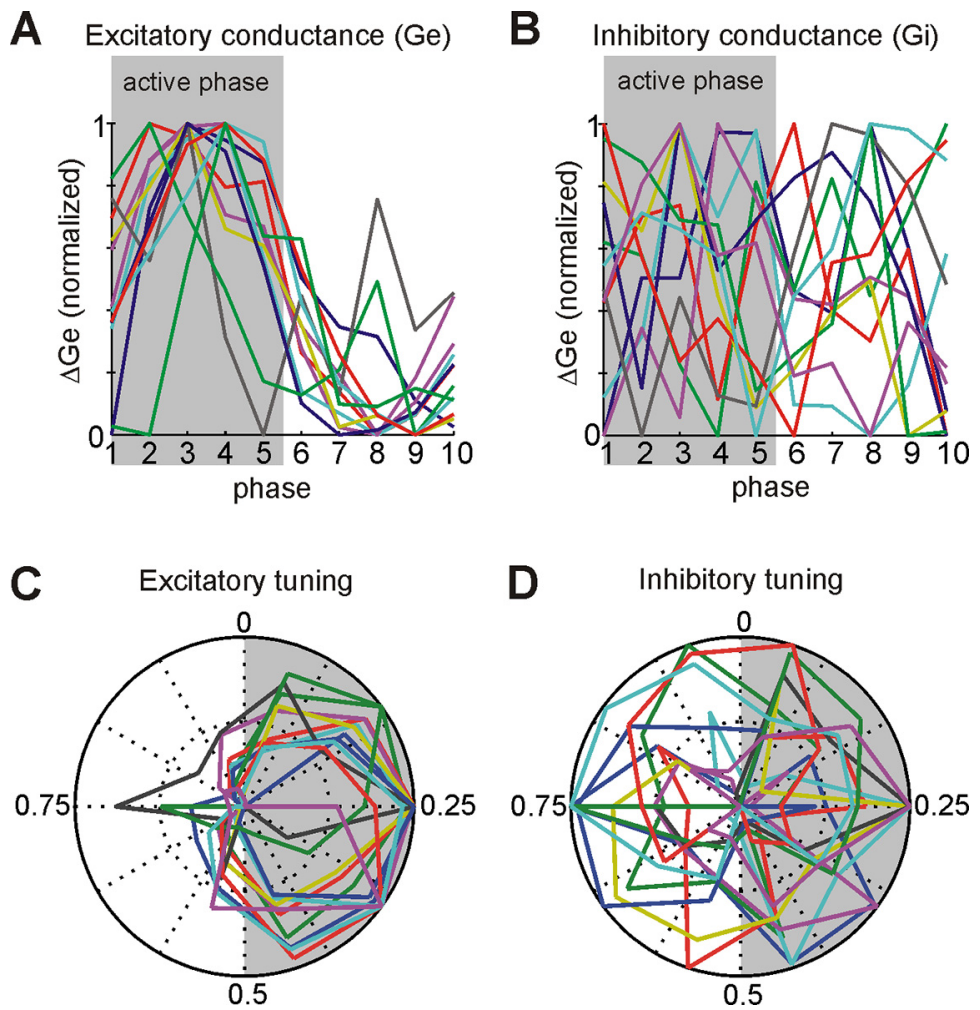

Figure 5. Chx10 neurons receive strong excitatory synaptic inputs during locomotor-like activity. Normalized excitatory $(\boldsymbol{A})$ and inhibitory $(\boldsymbol{B})$ decomposed conductances are shown for 12 Chx10 neurons in a normalized locomotor cycle in which bins 1-5 indicate the in-phase ventral root activity, whereas bins 5-10 indicate the out-of-phase ventral root activity. Note that, although all cells show strong rhythmic excitatory conductances in phase with the ventral root, the inhibitory conductances show no clear phasing. The tuning of the excitatory conductances were sharp around the peak of the ventral root burst (phase value, 0.25$)(\boldsymbol{C})$, whereas the inhibitory showed no tuning at all (D).

\section{Membrane properties of $\mathrm{Chx} 10$ neurons are heterogeneous}

Membrane properties have been shown to contribute in various ways to rhythmicity in many rhythmic motor systems (Marder and Calabrese, 1996; Kiehn et al., 2000; Harris-Warrick, 2002; Grillner, 2003; Ramirez et al., 2004). We, therefore, characterized the membrane and firing properties of Chx10 cells as well as a number of active membrane properties that have been directly involved in promoting rhythmicity.

Membrane properties were measured in 171 Chx10-GFP neurons in transverse section $(n=57)$, in horizontal section $(n=11)$, and in the dorsal horn-removed preparation $(n=103)$. There were no apparent differences between the measurements taken in these preparations. Membrane properties were measured in control conditions with intact synaptic connections. Since NMDA and 5-HT (and in some cases dopamine) were used to activate locomotor-like activity, we measured the effects these drugs had on the basic membrane properties in preparations with intact synaptic properties. In the presence of locomotion-inducing drugs, most membrane properties remained the same; however, there were some differences. Cells measured in NMDA/5-HT/ dopamine had a lower resistance $(849 \pm 65 \mathrm{M} \Omega ; n=74)$ than control cells ( $1614 \pm 158 \mathrm{M} \Omega ; n=68 ; p<0.00001)$. Cells also had a higher rheobase ( $35 \pm 4 \mathrm{pA}$ in drugs, $n=68 ; 22 \pm 3 \mathrm{pA}$ in control, $n=68$; $p<0.005)$ and a shorter membrane time constant $\tau$ (36 $\pm 3 \mathrm{~ms}$ in drugs, $n=71 ; 49 \pm 4 \mathrm{~ms}$ in control, $n=67$; $p<0.005)$. All other properties remained the same. Overall, these measurements show that the Chx10 cells, when embedded in the intact network, become less electrically compact, likely be- cause of a shunt attributable to synaptic input and/or opening of ion, ligand-gated, and voltage-gated channels (Raastad et al., 1998) either as a direct or indirect action of NMDA and/or 5-HT.

To further characterize the Chx10 neurons, we tested their responses to depolarizing current pulse injections. This protocol revealed the following four major categories of cells: (1) cells that fired throughout the current injection, (2) cells that showed a delayed firing, (3) cells that showed phasic firing, and (4) single spiking cells. These categories have previously all been described in studies of rodent interneurons in the dorsal and ventral horn (Hochman et al., 1997; Prescott and De Koninck, 2002; Szûcs et al., 2003; Ruscheweyh et al., 2004). (1) Cells that fired throughout the current injection represented the majority (74 of 171). These cells could be divided into two groups: tonic and burst-tonic. The tonic firing neurons were the most common $(n=62)$ and fired tonically throughout the current step with a slow adaptation in firing frequency (Fig. $\left.6 A_{1}\right)$. Burst-tonic firing neurons $(n=12)$ were characterized by a depolarization after the first spike in lowamplitude current steps and a burst of two action potentials, with a markedly higher instantaneous frequency than seen between the following spikes, in high-amplitude current steps (Fig. 6 $A_{2}$ ). (2) Delay firing neurons $(n=9)$ had a lag between the depolarization in response to the step and the first spike (Fig. $6 B$ ). In higher current amplitude steps, a first spike would appear and there would be a small delay before the firing would continue. (3) Phasic firing neurons could be divided into two categories: initial burst and "chattering." Initial burst neurons (Fig. $\left.6 C_{1}\right)(n=21)$ 
A

1 tonic

tonic
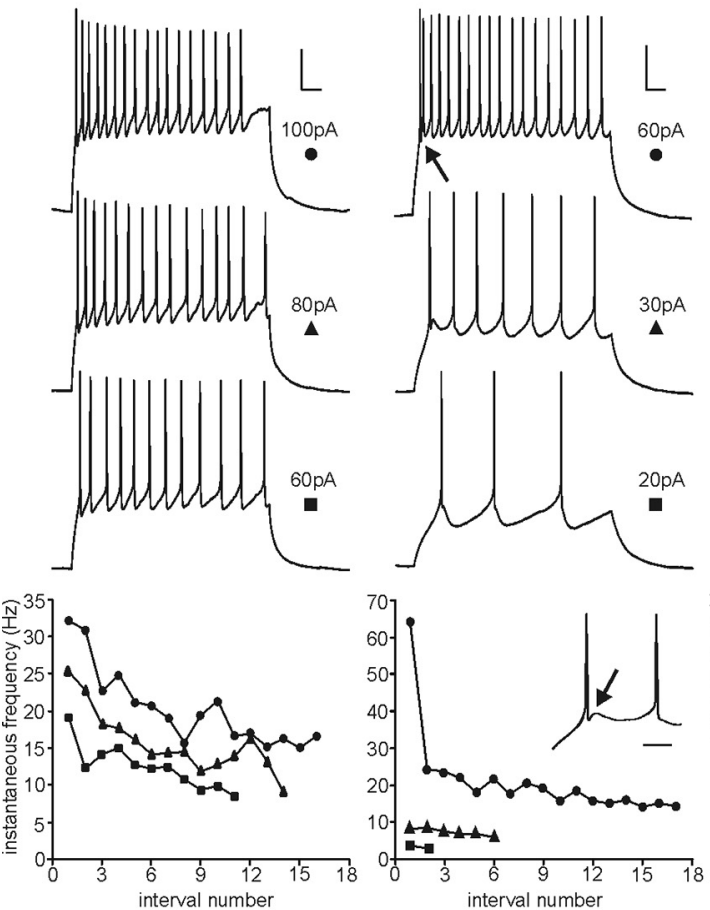

C

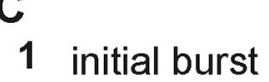

\section{phasic}

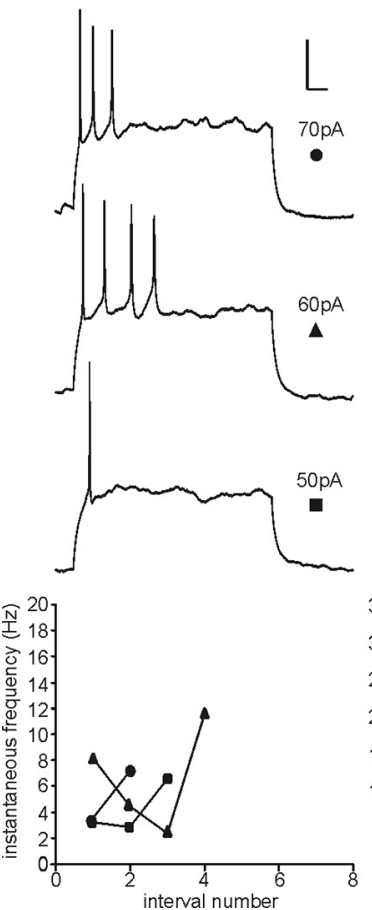

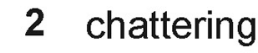

\section{B delay}
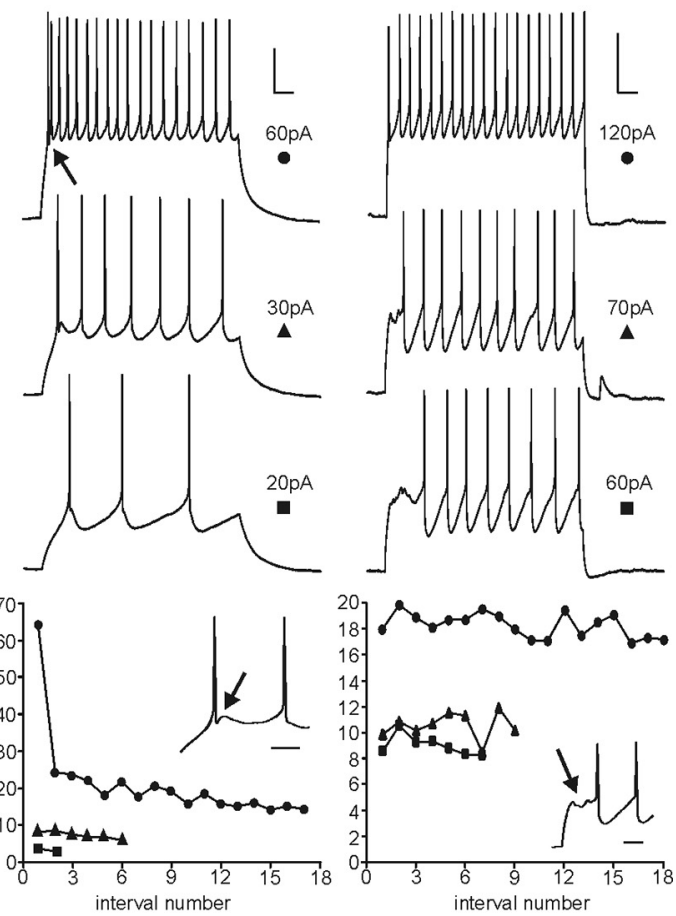

D single spike
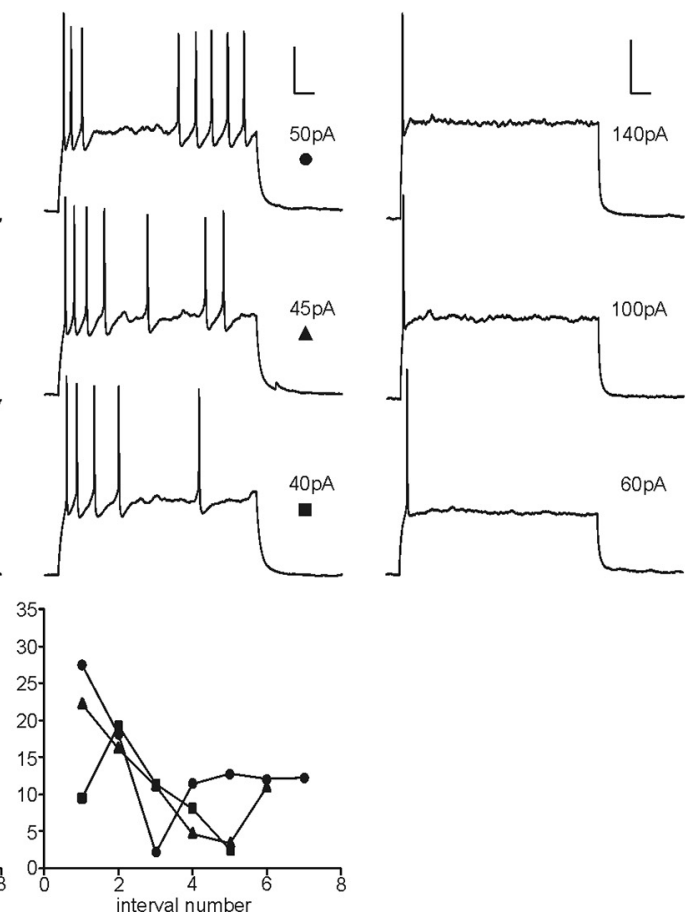

Figure 6. Firing properties of Chx10 neurons. Chx10 neuronal firing can be organized into four major firing categories based on their responses to square current pulses: tonic $(\boldsymbol{A})$, delay $(\boldsymbol{B})$, phasic $(\boldsymbol{C})$, and single-spike firing $(\boldsymbol{D})$. The tonic firing cells were further divided into tonic $\left(A_{1}\right)$ and burst-tonic $\left(A_{2}\right)$ firing neurons, whereas the phasic firing neurons were divided into initial burst $\left(\boldsymbol{C}_{1}\right)$ and chattering firing neurons $\left(\boldsymbol{C}_{2}\right)$. The values to the right of the intracellular traces indicate current step amplitude. The arrow in the top trace of $\boldsymbol{A} \mathbf{2}$ points to the burst of two action potentials seen in burst-tonic neurons. Frequency-current graphs are shown below the intracellular traces. Note the depolarizing hump that follows the first spike in the burst-tonic firing cells in $\boldsymbol{B}$ (arrow in inset) and the delayed onset of firing in $\boldsymbol{C}$ (arrow in inset). Calibration: Inset, $50 \mathrm{~ms}$; vertical (all), $20 \mathrm{mV}$; horizontal (all other), $100 \mathrm{~ms}$.

fired one to five action potentials only in the beginning of the step. Chattering neurons (Fig. $\left.6 C_{2}\right)(n=33)$ fired irregularly, on and off, during the current steps. (4) Last, there were cells that fired with only a single spike (Fig. $6 D)(n=27)$. The remaining seven neurons were tested only during drug-induced locomotion and could not be classified because of the rhythmic activity.

There were few significant differences in basic membrane properties between these types of cells (Table 1). Burst cells had the lowest $R_{\text {in }}$ and highest $C_{\mathrm{m}}$, indicating that they were the largest cells. Single-spike cells had the shortest membrane time constant and highest rheobase, whereas tonic cells had the longest membrane time constant and lowest rheobase. However, rhythmogenic membrane properties did show a relationship to these firing properties (see next section). All of these categories of Chx10 neurons contained cells that were active during rhythmic activity with no obvious preference for any category.

We also characterized potential rhythmogenic properties of Chx10 neurons in some detail. In particular, subsets of neurons were found to have postinhibitory rebound (PIR) (Fig. 7A), "sag" responses (Fig. $7 B$ ), and persistent inward currents (PICs) (Fig. 7C). PIR is related to deactivation of $I_{\mathrm{h}}$ and/or a transient calcium current, $I_{\mathrm{T}}$. Sag responses are related to the activation of a hyperpolarization activated cationic current, $I_{\mathrm{h}}$. PICs are seen as negative slope regions in the $I-V$ curve and are related to a persistent sodium current and/or activation of a persistent calcium current. These properties were correlated to some of the observed firing categories. A pronounced postinhibitory rebound depolarization with or without action potentials (Fig. 7A) was seen in every firing type but occurred most often in tonic firing neurons (Fig. $7 D$, percentage in second column). A sag response during hyperpolarizing steps in current clamp (Fig. $7 B$ ) was present in a large proportion of tonic (74\%) and burst-tonic (67\%) neurons, but was also seen to a lesser extent in all other firing types (27-44\%) (Fig. 7D). PICs were present in most cells tested (53 of 71) but were much smaller (mean peak current, $11 \pm 1 \mathrm{pA}$ ) than what has been reported in other ventrally located cells (Tazerart et al., 2007, 2008; Theiss et al., 2007; Zhong et al., 2007; Ziskind-Conhaim et al., 2008). However, our experiments were performed in the absence of any potassium channel blockers, and therefore active potassium con- 
Table 1. Comparison of membrane properties among different firing types

\begin{tabular}{|c|c|c|c|c|c|c|}
\hline & $\begin{array}{l}\text { Tonic } \\
(n=62)\end{array}$ & $\begin{array}{l}\text { Burst-tonic } \\
(n=12)\end{array}$ & $\begin{array}{l}\text { Delay } \\
(n=9)\end{array}$ & $\begin{array}{l}\text { Initial burst } \\
(n=21)\end{array}$ & $\begin{array}{l}\text { Chattering } \\
(n=33)\end{array}$ & $\begin{array}{l}\text { Single spike } \\
(n=27)\end{array}$ \\
\hline$V_{m}(m V)$ & $-47 \pm 1$ & $-51 \pm 1$ & $-49 \pm 2$ & $-48 \pm 2$ & $-51 \pm 1$ & $-49 \pm 2$ \\
\hline$R_{\text {in }}(\mathrm{pA})$ & $1590 \pm 146$ & $557 \pm 79^{*}$ & $986 \pm 212$ & $1234 \pm 263$ & $903 \pm 119$ & $849 \pm 149$ \\
\hline$\tau(\mathrm{ms})$ & $55 \pm 3^{*}$ & $37 \pm 3$ & $36 \pm 8$ & $35 \pm 4$ & $31 \pm 4$ & $18 \pm 3^{*}$ \\
\hline$C(\mathrm{pF})$ & $45 \pm 3$ & $73 \pm 6^{*}$ & $43 \pm 8$ & $40 \pm 4$ & $41 \pm 4$ & $23 \pm 2$ \\
\hline Peak outward current (pA) & $1472 \pm 75$ & $1870 \pm 202$ & $1739 \pm 314$ & $1483 \pm 110$ & $1398 \pm 118$ & $1403 \pm 149$ \\
\hline Peak inward current (pA) & $-1249 \pm 32$ & $-1859 \pm 162$ & $-1111 \pm 259$ & $-1002 \pm 97$ & $-1016 \pm 84$ & $-805 \pm 73$ \\
\hline Rheobase (pA) & $14 \pm 2^{*}$ & $23 \pm 2$ & $34 \pm 11$ & $30 \pm 5$ & $41 \pm 5$ & $80 \pm 16^{*}$ \\
\hline Spike height (mV) & $53 \pm 1$ & $59 \pm 2$ & $47 \pm 4$ & $47 \pm 2$ & $47 \pm 2$ & $44 \pm 2$ \\
\hline Overshoot (mV) & $19 \pm 1$ & $24 \pm 2$ & $14 \pm 4$ & $14 \pm 1$ & $11 \pm 2$ & $6 \pm 2$ \\
\hline Threshold (mV) & $-42 \pm 0.5$ & $-41 \pm 1$ & $-40 \pm 4$ & $-40 \pm 1$ & $-40 \pm 1$ & $-41 \pm 1$ \\
\hline
\end{tabular}

Values represent mean $\pm \mathrm{SE}$.

*Significance at $p<0.05$.

ductances may partially mask the PIC. The onset of the PIC occurred at $-53 \pm 1$ $\mathrm{mV}$, and the peak of the PIC was at $-36 \pm$ $1 \mathrm{mV}$, similar to has been reported for other cell types. PICs were found in all cell types with the exception of delay neurons (Fig. 7D). Rhythmicity during locomotorlike activity was not clearly related to the expression of $I_{\mathrm{h}}$, PIR, or PICs, as both rhythmic and nonrhythmic cell populations contained cells with and without each of these properties.

We conclude from these studies that the Chx10 cells have heterogeneous electrophysiological, firing, and rhythmogenic properties. Although these properties may provide distinguishable electrical fingerprints to subpopulations of Chx10 neurons, they do not separate cells into nonrhythmic and rhythmically active cells.

\section{Excitatory coupling among Chx10 cells is sparse}

Experiments in spinal excitatory CPG neurons in lamprey (Cangiano and Grillner, 2005) and tadpole (Li et al., 2009) and brainstem CPG neurons in mammals (Rekling et al., 2000) have suggested synaptic and/or electrical coupling as a means of synchronizing cellular firing. In early postnatal animals, electrical coupling is also a pronounced phenomenon both between motor neurons (Chang et al., 1999; Kiehn and Tresch, 2002; Tresch and Kiehn, 2002) and interneurons (Hinckley and Ziskind-Conhaim, 2006; Wilson et al., 2007). Since we saw cells firing in the same phase of the locomotor cycle and observed a strong excitatory input to Chx10 neurons in their active phase, we tested for synaptic and electrical coupling between Chx10 neurons. This was done using dual recordings from Chx10 neurons both in slices and in the dorsal horn-removed preparations. Of 4 pairs in the slices and 26 pairs in the dorsal horn-removed preparation, we were not able to demonstrate electrical or synaptic coupling (Fig. 8), either in cells that responded with similar spiking properties (Fig. $8 \mathrm{~A}, \mathrm{C}$ ) or in cells that had different spiking properties (Fig. $8 B, D$ ). We also recorded from seven pairs of cells in which

D neurons.

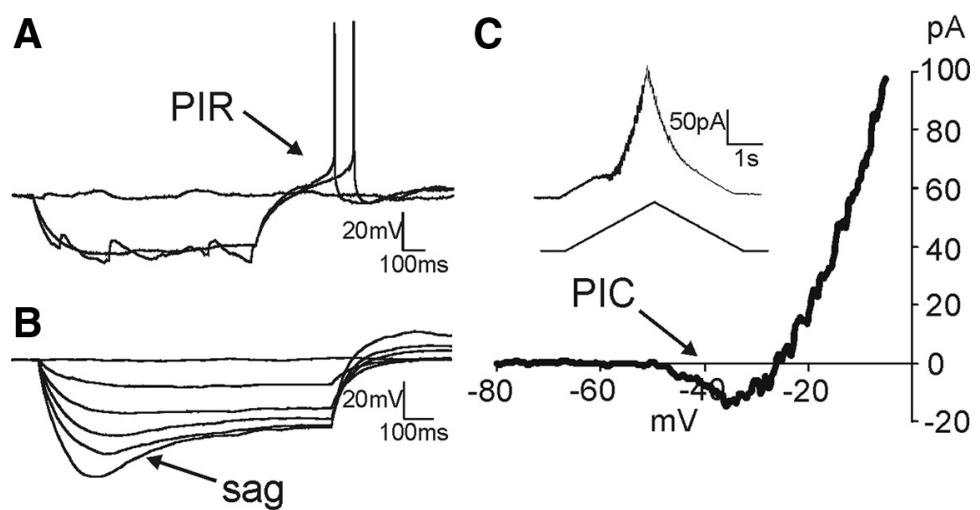

\begin{tabular}{|c|c|c|c|}
\hline Firing type & PIR & sag & PIC \\
\hline tonic & $42 \%$ & $74 \%$ & $89 \%$ \\
\hline tonic-burst & $8 \%$ & $67 \%$ & $100 \%$ \\
\hline delay & $22 \%$ & $44 \%$ & $0 \%$ \\
\hline initial burst & $29 \%$ & $38 \%$ & $86 \%$ \\
\hline chattering & $15 \%$ & $27 \%$ & $85 \%$ \\
\hline single & $11 \%$ & $15 \%$ & $27 \%$ \\
\hline
\end{tabular}

Figure 7. Chx10 neurons display a variety of rhythmogenic cellular properties. $\boldsymbol{A}$, Example of Chx10 neuron displaying PIR firing after hyperpolarizing current steps. $\boldsymbol{B}$, Example of $C h \times 10$ neuron displaying sag response, indicative of $I_{h}$, in response to hyperpolarization. C, PIC with pronounced negative slope region in the $I-V$ curve in a Chx10 neuron in response to a depolarizing slow voltage ramp. The displayed current is leak-subtracted. The inset shows voltage ramp and response before leak subtraction. D, Chart showing the incidence of PIR, sag, and PIC in the six firing categories depicted in Figure 6 of Chx10

both cells were rhythmically active during locomotion. An example of a pair in which both cells fired tonically is shown in Figure $8 A$, and an example in which cells were of two different firing types (here, chattering and single spike) is shown in Figure $8 B$. Although all of these cells were rhythmically active in the same locomotor phase (Fig. $8 A_{2}, B_{2}$ ), neither chemical nor electrical coupling was evident (Fig. $8 C, D$ ).

These studies show that, under our experimental conditions with intact synaptic connectivity, even in the relatively intact dorsal horn-removed preparation, excitatory coupling among Chx10 neurons is sparse or not easy to demonstrate. 

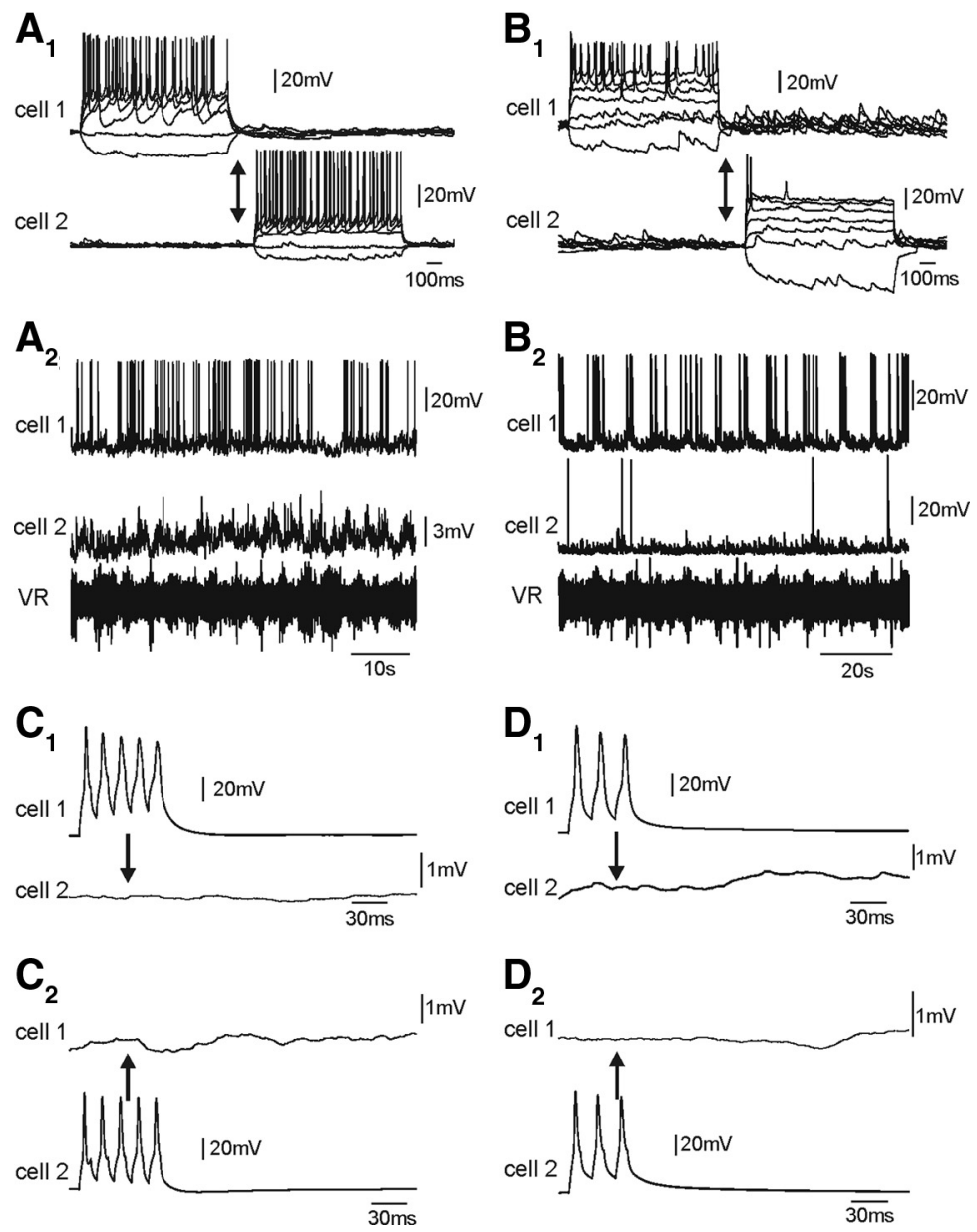

Figure 8. Chx10 neurons are sparsely synaptically and electrically coupled to other Chx10 neurons. $A_{1}$, Current step files of paired recordings of two Chx10 neurons with the same firing properties (tonic firing). Note that there is no sign of either synaptic or electrical coupling. $\boldsymbol{A}_{2}$, Same neurons as in $\boldsymbol{A}_{1}$ during locomotor-like activity at resting potentials (no currents injected). Neurons are rhythmically active in phase with each other. $\boldsymbol{B}_{1}$, Paired recordings from a phasic $\mathrm{Ch} \times 10$ neuron and a single spike firing $\mathrm{Chx} 10$ neuron. There is no sign of either synaptic or electrical coupling. $\boldsymbol{B}_{2}$, Paired recordings from the same neurons shown in $\boldsymbol{B}_{1}$ during locomotor-like activity. Both cells are rhythmically active in phase with each other. $C_{1}, D_{1}$, Average response of cell 2 to 50 trials of current-evoked spikes in cell 1. Cell 1 and cell 2 in $\boldsymbol{C}$ correspond to the cells shown in $\boldsymbol{A}$ and $\boldsymbol{D}$ corresponds to $\boldsymbol{B}$. $\boldsymbol{C}_{2}, \boldsymbol{D}_{2}$, Average response of cell 1 to 50 trials of current-evoked spikes in cell 2.

\section{Chx10 neuronal morphology reveals local and long-range projecting classes of neurons}

Anatomical and electrophysiological studies have suggested that Chx10 cells should project to motor neurons (Al-Mosawie et al., 2007; Crone et al., 2008) and commissural interneurons (Crone et al., 2008). Moreover, retrograde labeling studies have shown that only a minor percentage of Chx10 cells were backlabeled from more than one segment away (Lundfald et al., 2007). Moreover, the Chx10 homologues in zebrafish, called Alx neurons, have been shown to contact motor neurons through both chemical and electrical synapses (Kimura et al., 2006). Therefore, it was of interest to look at the anatomical projection patterns of individual Chx10 neurons in the mouse. This was done in both slice and dorsal horn-removed preparations. Patched cells were biocytin-filled for at least $20 \mathrm{~min}(20-90 \mathrm{~min})$ and incubated for at least $30 \mathrm{~min}$ before fixation to increase filling of distinct processes. In transverse slice, we observed Chx10 neurons to have a bifurcating axon that traveled toward the area of the medial and lateral motor nuclei crossing the area where commissural interneurons are located $(n=3)$ (Eide et al., 1999; Stokke et al., 2002; Nissen et al., 2005, Quinlan and Kiehn, 2007) (Fig. 9A), a single axon that appeared to terminate near where the commissural interneurons and/or medial motor neurons are located $(n=1)$, or processes that remained right around the soma of the labeled cell $(n=$ 1 ). This projection pattern is similar to what has been observed after intracellular fills in Lhx3 mice, in which one-half of the Lhx3positive cells are Chx10 neurons (Lundfald et al., 2007). In the dorsal horn-removed preparation, we were able to study the longitudinal projections of Chx10 neurons $(n=22)$. Using this preparation, we observed a great variety in the extent of projections that could not be seen in slices. Many of the filled cells $(n=14)$ appeared to be local interneurons with relatively short and compact dendrites and axonal projections that remained near the cell body (Fig. 9B). These cells all had dendrites oriented in the medial-lateral plane and projected into lamina VII where many locomotor-related neurons including commissural neurons are located. Cells with long ascending and/or descending (Fig. 9C-E) axons were also reconstructed $(n=8)$. These cells had axons that traveled laterally and then turned in the rostral, the caudal, or both rostral and caudal directions. Axonal branches of these cells ended in laminae VII/VIII and/or in the motor nuclei. The axons of all the filled Chx10 neurons remained strictly ipsilateral. Dye coupling between filled Chx10 neurons and unfilled Chx10 neurons or non-Chx10 neurons was never observed.

The intracellular fills show that the Chx10 neurons axonal branching pattern is compatible with them having effects on diverse populations of spinal neurons, which may include both motor neurons and commissural interneurons.

\section{Discussion}

Our study provides a report of V2a interneurons in a synaptically intact network in the rodent spinal cord. We demonstrate that many Chx10 interneurons are rhythmically active with either flexor-related or extensor-related activity along the length of the lumbar spinal cord. The rhythmicity of the Chx10 neurons is likely driven by synaptic inputs, as the Chx10 neurons received mostly in-phase excitatory input, were apparently not interconnected, and membrane properties showed no clear relation to rhythmicity. Our data are compatible with the Chx10 cells constituting groups of neurons involved in the regulation of leftright alternation and ipsilateral motor neuron firing in the rodent locomotor CPG.

\section{Chx10-GFP mouse is useful for studying V2a neurons}

In all experiments, we used a Chx10-EGFP transgenic mouse strain to visually identify Chx10 interneurons for electrophysiological recordings. The GFP expression in this mouse strain has been reported to be specific to Chx10-expressing neurons using in situ hybridization and to have a pattern of expression similar to the Chx10 expression in the brain (MMRC) (Gong et al., 2003). 
Here, we show that GFP-positive cells located in the ventral horn also express the Chx10 protein, whereas GFP-positive cells in the dorsal horn do not express Chx10. Similar ectopic expression of GFP in neurons not expressing the protein of interest has been reported in other GFPexpressing mice used for locomotor studies (cf. Hinckley et al., 2005; Wilson et al., 2005, 2007; Hinckley and ZiskindConhaim, 2006). Since the dorsal population has a weaker expression of GFP and is anatomically separated from the Chx10positive neurons in the ventral spinal cord, we were able to reproducibly target Chx10 neurons both in slices and in the dorsal horn-removed preparation without having to rely on secondary characteristics of the neurons (cf. Hinckley et al., 2005; Wilson et al., 2005, 2007; Hinckley and Ziskind-Conhaim, 2006).

\section{Chx10 rhythmicity}

The position of the Chx10 neurons deep in the cord prevented visual patching in the intact cord. However, by removing the dorsal horn by horizontal sectioning along the length of the lumbar cord, we obtained visual access to the entire Chx10 population in the lumbar cord in a preparation that was still able to produce a rhythmic motor output. The frequency of drug-induced locomotor activity observed in this preparation (mean, $0.17 \mathrm{~Hz}$ ) is in the lower range of what is observed before removal of the dorsal horn (mean, $0.24 \mathrm{~Hz}$ ) or previously reported for druginduced locomotor activity in the mouse (Kullander et al., 2003; Crone et al., 2008, 2009). Under these conditions, approximately two-thirds of Chx10 neurons were rhythmically active during drug-induced locomotor-like activity, as seen by rhythmic spiking or membrane potential oscillations. In flexor-dominated segments (L2-L3) and extensor dominated segments (L4-L5), most rhythmically active cells were active in phase with the ipsilateral segmental root. However, in all segments, there were also cells active out of phase with ipsilateral segmental root. Most cells firing action potentials at resting membrane potential were rhythmic during locomotor-like activity (25 of 38 ), with firing centered around phase values of 0.25 or 0.75 , which corresponds to the peak activity in either the ispilateral or contralateral segmental ventral roots. This timing of rhythmic spike activity in the Chx10 population along the cord is corresponding to what is found for L2 Chx10 neurons described in an accompanying paper (Zhong et al., 2010). Notably, the percentage of rhythmically active spiking Chx10 neurons in the two studies is comparable at low locomotor frequencies $(\sim 40 \%)$. We were unable to induce locomotor activity with middle to high frequencies possibly because the dorsal horn was removed along the entire lumbar cord [unlike the study by Zhong et al. (2010), in which the dorsal horn was removed only over L2]. We could therefore not determine whether a higher percentage of cells would be active at higher
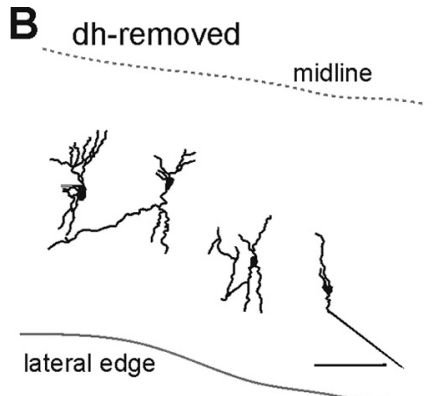

D dh-removed

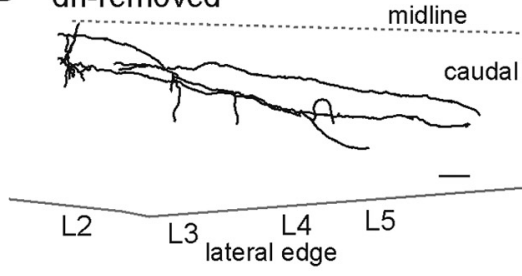

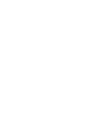

Figure 9. Chx10 neurons show variable cell morphology and axonal projections. $\boldsymbol{A}$, Examples of Chx10 neurons recorded in ocated, and bifurcate, possibly to the medial and lateral motor pools. $\boldsymbol{B}$, Neighboring local Chx10 neurons recorded in dorsal horn-removed preparation. All neurons have processes that remain very local and appear columnar in orientation. $\boldsymbol{C}$, Example of an neuron recorded in $\mathrm{L} 2$ in a dorsal horn-removed preparation. The dendrites remain very local. However, the axon travels laterally Chx10 neuron recorded in a dorsal horn-removed preparation. The dendrites are all local and the axon first travels laterally, and

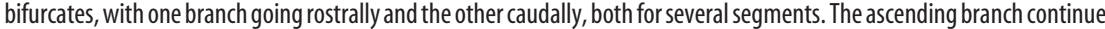
to run close to the lateral edge of the spinal cord. In the segment of the soma, there is a collateral that turns near to the soma. In two other caudal segments, an axon collateral can be seen turning in more medially and branching.

locomotor frequencies as observed in the accompanying paper (Zhong et al., 2010). However, the present findings, together with findings in the accompanying paper, are compatible with both flexor- and extensor-related Chx10 neurons providing in-phase synaptic drive to segmental flexor- and extensor-related motor neurons that are found in both L2/L3 and L4/L5 (anatomy) (Endo and Kiehn, 2008). The activity of the flexor- and extensorrelated Chx10 neurons observed along the lumbar cord is also suited to drive the dual segmental commissural interneuron system involved in controlling left-right alternation (Quinlan and Kiehn, 2007; Crone et al., 2008). This notion is further supported by the segmental axonal projection pattern of many Chx10 neurons in lamina VII and/or the motor nucleus, as revealed by intracellular staining.

The presence of both flexor- and extensor-related interneurons in the same segment is not unique to Chx10 neurons. Renshaw cells (Nishimaru et al., 2006) and commissural interneurons (Butt et al., 2002) also show both flexor- and extensorrelated firing in the same segment. The diversity of Chx10 firing therefore matches the commissural interneuron firing and suggests that Chx10 interneurons may be organized into 
flexor- and extensor-related modules in a single segment (any segment L2-L5). Each segmental module contains Chx10 neurons that will be out of phase with the antagonist module. The majority of Chx10 neurons, including those out of phase with the ipsilateral segmental root, likely project to commissural interneurons or motor neurons in the same segment because ablation of Chx10 neurons does not disrupt flexor-extensor alternation (Crone et al., 2008), and the majority of filled Chx10 neurons were local interneurons.

Based on the ablation experiments (Crone et al., 2008), we also proposed that Chx10 neuron activity is not the primary source of rhythm generation. Rather, Chx10 activity is driven by convergent in-phase excitatory input from rhythm-generating CPG neurons. The synaptic conductance analysis performed here provides support to this notion since Chx10 cells receive predominantly rhythmic synaptic excitation during locomotor-like activity. This rhythmic excitation was likely not generated from recurrent synaptic excitation among Chx10 cells, as we were unable to reveal synaptic connectivity in a large number of dual recordings in a synaptically intact network, regardless of rhythmicity or combination of firing patterns displayed. Together, these findings suggest that external synaptic input alone recruits Chx10 neurons into the locomotor rhythm. In this sense, Chx10 neurons differ significantly from identified excitatory spinal CPG interneurons in the tadpole and lamprey, which have been shown to be interconnected chemically (Cangiano and Grillner, 2005; Li et al., 2009).

In the present study, we did not find any signs of electrical connections between Chx10 neurons in a synaptically intact network. This finding is in sharp contrast to what is found by Zhong et al. (2010), who showed that $30 \%$ of the tonic Chx10 neurons were mutually electrically coupled in slices with all synaptic inputs blocked. We do not have a clear explanation for these differences. It is possible that blocking all synaptic inputs will make the cell more electrically compact and therefore more prone to see electrical coupling. Alternatively, the differences could be attributable to differences in the mice strain used $[\mathrm{FVB} / \mathrm{N}$ Swiss Webster (this study) vs C57/black6 (Zhong et al., 2010)] or the fact that this study uses mice in which the transgene is randomly inserted in the genome, whereas Zhong et al. (2010) use mice in which the GFP insertion makes one of the Chx10 alleles nonfunctional.

A significant proportion (approximately one-third) of Chx10 cells did not display rhythmic activity at all. This proportion is similar to what has been shown for all ventral interneuronal populations sampled during drug-induced locomotion (Kiehn et al., 1996; Tresch and Kiehn, 1999; Raastad et al., 2000; Butt et al., 2002; Wilson et al., 2005, 2007; Hinckley and Ziskind-Conhaim, 2006; Zhong et al., 2006) with the exception of one report in which almost $90 \%$ of $\mathrm{Hb} 9$-positive neurons were rhythmically active (Hinckley et al., 2005). It is possible that nonrhythmic Chx10 cells are not engaged directly in rhythmic CPG activity or that they are recruited under different speeds or patterns of locomotor activity. Another possibility is that the nonrhythmic Chx10 neurons constitute a separate population of Chx10 neurons needed to mediate sensory-evoked locomotor-like activity. Thus, in the absence of spinal Chx10 cells, dorsal root stimulation fails to evoke locomotor-like activity (Crone et al., 2008). Such sensory gating cells do not need to be rhythmically active to mediate their effect on the locomotor network. We have not been able to test this possibility directly in the present series of experiments because we have removed the dorsal horn.

\section{Membrane properties of Chx10 neurons are heterogeneous, not related to rhythmicity, and shared with other spinal interneurons}

The analysis of firing and membrane properties showed that the Chx10 population was electrophysiologically heterogeneous. All the firing types described here have been described by others (Hochman et al., 1997; Prescott and De Koninck, 2002; Szûcs et al., 2003; Ruscheweyh et al., 2004) and correspond well with those described in the accompanying paper. Although we were able to identify distinct Chx10 interneuron firing patterns that also showed correlations to rhythmic membrane properties (PIC, PIR, and $I_{\mathrm{h}}$ ), it was not obvious that these membrane properties contributed specifically to Chx10 neuron rhythmicity since cells of all firing types were capable of being rhythmically active during locomotion. The membrane properties of the Chx10 population may therefore play a less significant role in recruiting the neurons into the network than the synaptic inputs that they receive. Moreover, rhythmogenic membrane properties (Butt et al., 2002; Wilson et al., 2005; Hinckley and Ziskind-Conhaim, 2006; Zhong et al., 2006) have been identified in other populations of rodent spinal interneurons, suggesting that they themselves do not provide a distinct electrophysiological signature. Having said this, however, it is not impossible that the distinct firing patterns observed here may correspond to uniquely molecularly defined populations since the V2a population can be further subdivided based on expression on other transcription factors than Chx10 (Dougherty et al., 2009).

\section{Comparison of Chx10 neurons with other ipsilaterally projecting excitatory vertebrate CPG interneurons}

Our morphology studies show that Chx10 neurons, although strictly ipsilateral, vary greatly in axonal projections. In this sense, they differ from their molecular homolog, the Alx neurons, in the zebrafish, which, like ipsilateral excitatory CPG neuron counterparts in the tadpole and lamprey, have descending axons (Buchanan and Grillner, 1987; Buchanan et al., 1989; Kimura et al., 2006; McLean et al., 2008; Li et al., 2009). Most of the Chx10 cells have short segmental axons, whereas only a minor fraction has long projecting axons. In contrast, the Chx10 neuronal projections appear to be similar to those recently seen in excitatory, ipsilateral, lamina VII neurons in cat (Bannatyne et al., 2009). We propose that the short projecting Chx10 neurons are those involved in coordination of left-right alternation during locomotion (Crone et al., 2008). The coordinating role of the longprojecting Chx10 neurons found in the present study is less clear, but they could serve a role in coordinating motor neuron activities along the cord.

Another distinction from the lamprey and tadpole is that the descending excitatory interneurons in these animals are supposed to generate the rhythm, whereas this is clearly not the case for Chx10 neurons in the mouse spinal cord. In zebrafish, Alx neurons can be subdivided based on whether they are active during strong, fast locomotion or during slow, weak locomotion (Kimura et al., 2006). At higher locomotor speeds, new Alx neurons are recruited, whereas those active at slower speeds are actively inhibited (McLean et al., 2008). In the present study, we did not see any sign of speed-related recruitment of Chx10. However, the locomotor-like activity evoked in the dorsal horn-removed preparation was in the slow range, and, although most Chx10 cells were rhythmically active at this slow speed (Crone et al., 2009), it remains a possibility that the population of nonrhythmic cells that we observed may be active during stronger locomotor-like activity (Zhong et al., 2010). Together, our data 
lend support to the suggestion that the swimming and walking CPG, although sharing similarities in their organization, may display distinct differences (Kiehn, 2006).

\section{References}

Al-Mosawie A, Wilson JM, Brownstone RM (2007) Heterogeneity of V2derived interneurons in the adult mouse spinal cord. Eur J Neurosci 26:3003-3015.

Bannatyne BA, Liu TT, Hammar I, Stecina K, Jankowska E, Maxwell DJ (2009) Excitatory and inhibitory intermediate zone interneurons in pathways from feline group I and II afferents: differences in axonal projections and input. J Physiol 587:379-399.

Berg RW, Alaburda A, Hounsgaard J (2007) Balanced inhibition and excitation drive spike activity in spinal half-centers. Science 315:390-393.

Buchanan JT, Grillner S (1987) Newly identified "glutamate interneurons" and their role in locomotion in the lamprey spinal cord. Science 236:312-314.

Buchanan JT, Grillner S, Cullheim S, Risling M (1989) Identification of excitatory interneurons contributing to generation of locomotion in lamprey: structure, pharmacology, and function. J Neurophysiol 62:59-69.

Butt SJ, Kiehn O (2003) Functional identification of interneurons responsible for left-right coordination of hindlimbs in mammals. Neuron 38:953-963.

Butt SJ, Harris-Warrick RM, Kiehn O (2002) Firing properties of identified interneuron populations in the mammalian hindlimb central pattern generator. J Neurosci 22:9961-9971.

Cangiano L, Grillner S (2005) Mechanisms of rhythm generation in a spinal locomotor network deprived of crossed connections: the lamprey hemicord. J Neurosci 25:923-935.

Cepeda-Nieto AC, Pfaff SL, Varela-Echavarría A (2005) Homeodomain transcription factors in the development of subsets of hindbrain reticulospinal neurons. Mol Cell Neurosci 28:30-41.

Chang Q, Gonzalez M, Pinter MJ, Balice-Gordon RJ (1999) Gap junctional coupling and patterns of connexin expression among neonatal rat lumbar spinal motor neurons. J Neurosci 19:10813-10828.

Clarac F (2008) Some historical reflections on the neural control of locomotion. Brain Res Rev 57:13-21.

Cowley KC, Schmidt BJ (1994) Some limitations of ventral root recordings for monitoring locomotion in the in vitro neonatal rat spinal cord preparation. Neurosci Lett 171:142-146.

Crone SA, Quinlan KA, Zagoraiou L, Droho S, Restrepo CE, Lundfald L, Endo T, Setlak J, Jessell TM, Kiehn O, Sharma K (2008) Genetic ablation of V2a ipsilateral interneurons disrupts left-right locomotor coordination in mammalian spinal cord. Neuron 60:70-83.

Crone SA, Zhong G, Harris-Warrick R, Sharma K (2009) In mice lacking V2a interneurons, gait depends on speed of locomotion. J Neurosci 29:7098-7109.

Dougherty KJ, Kiehn O (2008) Characterization of excitatory V2a interneurons in the mammalian spinal cord. Soc Neurosci Abstr 34:374.13.

Dougherty KJ, Mendelsohn M, Han B, Jessell T, Kiehn O, Zagoraiou L (2009) Rhythmogenic properties and connectivity of a transcriptionally defined subset of ipsilateral excitatory interneurons in mouse spinal cord. Soc Neurosci Abstr 35:366.1.

Eide AL, Glover J, Kjaerulff O, Kiehn O (1999) Characterization of commissural interneurons in the lumbar region of the neonatal rat spinal cord. J Comp Neurol 403:332-345.

Endo T, Kiehn O (2008) Asymmetric operation of the locomotor central pattern generator in the neonatal mouse spinal cord. J Neurophysiol 100:3043-3054.

Gong S, Zheng C, Doughty ML, Losos K, Didkovsky N, Schambra UB, Nowak NJ, Joyner A, Leblanc G, Hatten ME, Heintz N (2003) A gene expression atlas of the central nervous system based on bacterial artificial chromosomes. Nature 425:917-925.

Gordon IT, Whelan PJ (2006) Deciphering the organization and modulation of spinal locomotor central pattern generators. J Exp Biol 209: 2007-2014.

Goulding M, Pfaff SL (2005) Development of circuits that generate simple rhythmic behaviors in vertebrates. Curr Opin Neurobiol 15:14-20.

Grillner S (2003) The motor infrastructure: from ion channels to neuronal networks. Nat Rev Neurosci 4:573-586.

Harris-Warrick RM (2002) Voltage-sensitive ion channels in rhythmic motor systems. Curr Opin Neurobiol 12:646-651.
Hinckley CA, Ziskind-Conhaim L (2006) Electrical coupling between locomotor-related excitatory interneurons in the mammalian spinal cord. J Neurosci 26:8477-8483.

Hinckley CA, Hartley R, Wu L, Todd A, Ziskind-Conhaim L (2005) Locomotor-like rhythms in a genetically distinct cluster of interneurons in the mammalian spinal cord. J Neurophysiol 93:1439-1449.

Hochman S, Garraway SM, Pockett S (1997) Membrane properties of deep dorsal horn neurons from neonatal rat spinal cord in vitro. Brain Res 767:214-219.

Iizuka M, Kiehn O, Kudo N (1997) Development in neonatal rats of the sensory resetting of the locomotor rhythm induced by NMDA and 5-HT. Exp Brain Res 114:193-204.

Jankowska E (2008) Spinal interneuronal networks in the cat: elementary components. Brain Res Rev 57:46-55.

Jessell TM (2000) Neuronal specification in the spinal cord: inductive signals and transcriptional codes. Nat Rev Genet 1:20-29.

Jordan LM, Liu J, Hedlund PB, Akay T, Pearson KG (2008) Descending command systems for the initiation of locomotion in mammals. Brain Res Rev 57:183-191.

Kiehn O (2006) Locomotor circuits in the mammalian spinal cord. Annu Rev Neurosci 29:279-306.

Kiehn O, Kjaerulff O (1996) Spatiotemporal characteristics of 5-HT and dopamine-induced rhythmic hindlimb activity in the in vitro neonatal rat. J Neurophysiol 75:1472-1482.

Kiehn O, Kjaerulff O (1998) Distribution of central pattern generators for rhythmic motor outputs in the spinal cord of limbed vertebrates. Ann N Y Acad Sci 860:110-129.

Kiehn O, Tresch MC (2002) Gap junctions and motor behavior. Trends Neurosci 25:108-115.

Kiehn O, Johnson BR, Raastad M (1996) Plateau properties in mammalian spinal interneurons during transmitter-induced locomotor activity. Neuroscience 75:263-273.

Kiehn O, Kjaerulff O, Tresch MC, Harris-Warrick RM (2000) Contributions of intrinsic motor neuron properties to the production of rhythmic motor output in the mammalian spinal cord. Brain Res Bull 53:649-659.

Kimura Y, Okamura Y, Higashijima S (2006) alx, a zebrafish homolog of Chx10, marks ipsilateral descending excitatory interneurons that participate in the regulation of spinal locomotor circuits. J Neurosci 26:5684-5697.

Kjaerulff O, Kiehn O (1996) Distribution of networks generating and coordinating locomotor activity in the neonatal rat spinal cord in vitro: a lesion study. J Neurosci 16:5777-5794.

Kullander K, Butt SJ, Lebret JM, Lundfald L, Restrepo CE, Rydström A, Klein R, Kiehn O (2003) Role of EphA4 and EphrinB3 in local neuronal circuits that control walking. Science 299:1889-1892.

Kuo JJ, Siddique T, Fu R, Heckman CJ (2005) Increased persistent $\mathrm{Na}^{+}$ current and its effect on excitability in motoneurons cultured from mutant SOD1 mice. J Physiol 563:843-854.

Li WC, Roberts A, Soffe SR (2009) Locomotor rhythm maintenance: electrical coupling among premotor excitatory interneurons in the brainstem and spinal cord of young Xenopus tadpoles. J Physiol 587:1677-1693.

Lundfald L, Restrepo CE, Butt SJ, Peng CY, Droho S, Endo T, Zeilhofer HU, Sharma K, Kiehn O (2007) Phenotype of V2-derived interneurons and their relationship to the axon guidance molecule EphA4 in the developing mouse spinal cord. Eur J Neurosci 26:2989-3002.

Marder E, Calabrese RL (1996) Principles of rhythmic motor pattern generation. Physiol Rev 76:687-717.

McCrea DA, Rybak IA (2008) Organization of mammalian locomotor rhythm and pattern generation. Brain Res Rev 57:134-146.

McLean DL, Masino MA, Koh IY, Lindquist WB, Fetcho JR (2008) Continuous shifts in the active set of spinal interneurons during changes in locomotor speed. Nat Neurosci 11:1419-1429.

Nishimaru H, Restrepo CE, Kiehn O (2006) Activity of Renshaw cells during locomotor-like rhythmic activity in the isolated spinal cord of neonatal mice. J Neurosci 26:5320-5328.

Nissen UV, Mochida H, Glover JC (2005) Development of projectionspecific interneurons and projection neurons in the embryonic mouse and rat spinal cord. J Comp Neurol 483:30-47.

O'Donovan MJ, Bonnot A, Mentis GZ, Arai Y, Chub N, Shneider NA, Wenner P (2008) Imaging the spatiotemporal organization of neural activity in the developing spinal cord. Dev Neurobiol 68:788-803.

Prescott SA, De Koninck Y (2002) Four cell types with distinctive mem- 
brane properties and morphologies in lamina I of the spinal dorsal horn of the adult rat. J Physiol 539:817-836.

Quinlan KA, Kiehn O (2007) Segmental, synaptic actions of commissural interneurons in the mouse spinal cord. J Neurosci 27:6521-6530.

Raastad M, Kiehn O (2000) Spike coding during locomotor network activity in ventrally located neurons in the isolated spinal cord from neonatal rat. J Neurophysiol 83:2825-2834.

Raastad M, Enríquez-Denton M, Kiehn O (1998) Synaptic signaling in an active central network only moderately changes passive membrane properties. Proc Natl Acad Sci U S A 95:10251-10256.

Ramirez JM, Tryba AK, Peña F (2004) Pacemaker neurons and neuronal networks: an integrative view. Curr Opin Neurobiol 14:665-674.

Rekling JC, Shao XM, Feldman JL (2000) Electrical coupling and excitatory synaptic transmission between rhythmogenic respiratory neurons in the preBötzinger complex. J Neurosci 20:RC113(1-5).

Rowan S, Cepko CL (2004) Genetic analysis of the homeodomain transcription factor Chx10 in the retina using a novel multifunctional BAC transgenic mouse reporter. Dev Biol 271:388-402.

Ruscheweyh R, Ikeda H, Heinke B, Sandkühler J (2004) Distinctive membrane and discharge properties of rat spinal lamina I projection neurones in vitro. J Physiol 555:527-543.

Stepien AE, Arber S (2008) Probing the locomotor conundrum: descending the "V" interneuron ladder. Neuron 60:1-4.

Stokke MF, Nissen UV, Glover JC, Kiehn O (2002) Projection patterns of commissural interneurons in the lumbar spinal cord of the neonatal rat. J Comp Neurol 446:349-359.

Szûcs P, Odeh F, Szokol K, Antal M (2003) Neurons with distinctive firing patterns, morphology and distribution in laminae V-VII of the neonatal rat lumbar spinal cord. Eur J Neurosci 17:537-544.

Tazerart S, Viemari JC, Darbon P, Vinay L, Brocard F (2007) Contribution of persistent sodium current to locomotor pattern generation in neonatal rats. J Neurophysiol 98:613-628.

Tazerart S, Vinay L, Brocard F (2008) The persistent sodium current generates pacemaker activities in the central pattern generator for locomotion and regulates the locomotor rhythm. J Neurosci 28:8577-8589.

Thaler J, Harrison K, Sharma K, Lettieri K, Kehrl J, Pfaff SL (1999) Active suppression of interneuron programs within developing motor neurons revealed by analysis of homeodomain factor HB9. Neuron 23:675-687.

Theiss RD, Kuo JJ, Heckman CJ (2007) Persistent inward currents in rat ventral horn neurones. J Physiol 580:507-522.

Tresch MC, Kiehn O (1999) Coding of locomotor phase in populations of neurons in rostral and caudal segments of the neonatal rat lumbar spinal cord. J Neurophysiol 82:3563-3574.

Tresch MC, Kiehn O (2002) Synchronization of motor neurons during locomotion in the neonatal rat: predictors and mechanisms. J Neurosci 22:9997-10008.

Whelan P, Bonnot A, O'Donovan MJ (2000) Properties of rhythmic activity generated by the isolated spinal cord of the neonatal mouse. J Neurophysiol 84:2821-2833.

Wilson JM, Hartley R, Maxwell DJ, Todd AJ, Lieberam I, Kaltschmidt JA, Yoshida Y, Jessell TM, Brownstone RM (2005) Conditional rhythmicity of ventral spinal interneurons defined by expression of the $\mathrm{Hb} 9$ homeodomain protein. J Neurosci 25:5710-5719.

Wilson JM, Cowan AI, Brownstone RM (2007) Heterogeneous electrotonic coupling and synchronization of rhythmic bursting activity in mouse $\mathrm{Hb} 9$ interneurons. J Neurophysiol 98:2370-2381.

Zar JH (1974) Biostatistical analysis. Edgewood Cliffs, NJ: Prentice-Hall.

Zhong G, Díaz-Ríos M, Harris-Warrick RM (2006) Intrinsic and functional differences among commissural interneurons during fictive locomotion and serotonergic modulation in the neonatal mouse. J Neurosci 26:6509-6517.

Zhong G, Masino MA, Harris-Warrick RM (2007) Persistent sodium currents participate in fictive locomotion generation in neonatal mouse spinal cord. J Neurosci 27:4507-4518.

Zhong G, Droho S, Crone S, Dietz S, Kwan AC, Webb WW, Sharma K, Harris-Warrick RM (2010) Electrophysiological characterization of the V2a interneurons and their locomotor-related activity in the neonatal mouse spinal cord. J Neurosci 30:170-182.

Ziskind-Conhaim L, Wu L, Wiesner EP (2008) Persistent sodium current contributes to induced voltage oscillations in locomotor-related hb9 interneurons in the mouse spinal cord. J Neurophysiol 100:2254-2264. 